\title{
Methylidyne Transfer as a Plausible Deactivation Pathway for Ynene Metathesis.
}

Takashi Kurogi*,† and Daniel J. Mindiola*,‡

${ }^{\dagger}$ Division of Applied Chemistry, Graduate School of Natural Science and Technology, Okayama University, 3-1-1 Tsushimanaka, Kita-ku, Okayama 700-8530, Japan.

\# Department of Chemistry, University of Pennsylvania, 231 South 34th Street, Philadelphia PA, 19104, United States.

\section{Table of Contents}

General Procedure

Synthesis of $\left[(\mathrm{PNP}) \mathrm{Nb}\left(\mathrm{CH}=\mathrm{CHCH}_{3}\right)\left(\eta^{2}-\mathrm{C}_{2} \mathrm{H}_{4}\right)(\mathrm{OAr})\right](2)$

S3-S4

NMR Spectroscopic Data of 2

S4-S5

Synthesis of $\left[(\mathrm{PNP}) \mathrm{Nb}\left(\eta^{3}-\mathrm{C}_{3} \mathrm{H}_{5}\right)(\mathrm{OAr})\right](3)$

S5-S6

NMR Spectroscopic Data of 3

S6-S7

NMR Monitoring Reaction of 1 with $\mathrm{C}_{2} \mathrm{H}_{4}$

S8-S10

NMR Monitoring Reaction of $1-{ }^{13} \mathrm{C}$ with $\mathrm{C}_{2} \mathrm{H}_{4}$

S11-S15

NMR Monitoring Reaction of ${ }^{2-13} \mathrm{C}$ under $\mathrm{N}_{2}$

Polymerization of 2-Norbornene by 1

S18-S19

NMR Monitoring Reaction of $1-{ }^{13} \mathrm{C}$ with 2-Norbornene

X-ray Crystallography

Crystallographic data of $\mathbf{2}$

Crystallographic data of $\mathbf{3}$ 


\section{General Procedure}

All operations were performed in an M. Braun glove box or using standard Schlenk techniques under a nitrogen atmosphere unless otherwise stated. Toluene and ${ }^{n}$ pentane were purchased from Fisher Scientific and purified and dried by passage through two columns of activated alumina and Q-5 drying agent in a Grubbs-type solvent system. All bulk solvents were kept over sodium and $4 \AA$ molecular sieves. Benzene- $d_{6}$ (Cambridge Isotope Laboratories) was dried and degassed over a potassium mirror prior to use. $4 \AA$ molecular sieves were activated under vacuum overnight at $200{ }^{\circ} \mathrm{C}$. (PNP) $\mathrm{Nb}(\mathrm{CH})(\mathrm{OAr})(\mathbf{1})$ and $(\mathrm{PNP}) \mathrm{Nb}\left({ }^{13} \mathrm{CH}\right)(\mathrm{OAr})(\mathbf{1 - 1 3} \mathbf{C})$ (from 99 atom\% ${ }^{13} \mathrm{C}$ iodomethane, Sigma-Aldrich) were prepared according to the reported procedure. ${ }^{1} 2$ Norbornene was purchased from TCI and distilled over sodium prior to use. $\mathrm{C}_{2} \mathrm{H}_{4}$ (UHP grade, 99.9\%) was purchased from Airgas. ${ }^{1} \mathrm{H},{ }^{13} \mathrm{C},{ }^{31} \mathrm{P}$, HSQC and HMBC NMR spectra were recorded on a Bruker AV-II $500 \mathrm{MHz}$ or AV-III $400 \mathrm{MHz}$ spectrometers. ${ }^{1} \mathrm{H}$ and ${ }^{13} \mathrm{C}$ NMR chemical shifts are reported referenced to the internal residual proton or carbon resonances of $\mathrm{C}_{6} \mathrm{D}_{6}(\delta=7.16 \mathrm{ppm}$ or $128.06 \mathrm{ppm}) .{ }^{31} \mathrm{P}$ NMR chemical shifts are reported with respect to external $\mathrm{H}_{3} \mathrm{PO}_{4}(\delta=0.0 \mathrm{ppm})$. Elemental analyses were performed by Midwest Microlab, Inc. 


\section{Synthesis of $\left[(\mathrm{PNP}) \mathrm{Nb}\left(\mathrm{CH}=\mathrm{CHCH}_{3}\right)\left(\eta^{2}-\mathrm{C}_{2} \mathrm{H}_{4}\right)(\mathrm{OAr})\right](2)$}

A $50 \mathrm{~mL}$ Schlenk tube was charged with a green solution of $1(270 \mathrm{mg}, 379 \mu \mathrm{mol})$ in toluene $(10 \mathrm{~mL})$. The headspace was evacuated and an atmosphere of $\mathrm{C}_{2} \mathrm{H}_{4}(1 \mathrm{~atm})$ was introduced at room temperature. The color of the mixture gradually turned from green to reddish brown. After stirring for 5 hours at room temperature, the reaction mixture was evaporated to dryness. The brown residue was dissolved in pentane $(5 \mathrm{~mL})$ and filtered through glass wool in a pipette. The brown filtrate was concentrated to ca. $2 \mathrm{~mL}$ and stored at $-35^{\circ} \mathrm{C}$ overnight to yield 2 as reddish brown crystals (231 mg, 301 $\mu \mathrm{mol}, 79 \%$ yield).

${ }^{1} \mathrm{H}$ NMR (400 MHz, Benzene- $d_{6}, 300 \mathrm{~K}$ ): $\delta 7.76$ (br, 2H, Ar-CH), 7.16 (overlapped with $\left.\mathrm{C}_{6} \mathrm{D}_{5} \mathrm{H}, 2 \mathrm{H}, \mathrm{Ar}-\mathrm{CH}\right), 7.03$ (br, $\left.1 \mathrm{H}, \mathrm{Ar}-\mathrm{CH}\right), 6.94$ (br, 1H, Ar-CH), 6.86-6.74 (m, overlapped, 3H, $\mathrm{Ar}-\mathrm{CH}$ ), 5.11 (br s, $2 \mathrm{H}, \mathrm{NbCH}=\mathrm{CHCH}_{3}$ and $\mathrm{NbCH}=\mathrm{CHCH}_{3}$ ), 3.27 (br, 1H, OAr- $\left.\mathrm{CH}\left(\mathrm{CH}_{3}\right)_{2}\right), 3.14$ (br, $\left.2 \mathrm{H}, \mathrm{NbC}_{2} \mathrm{H}_{4}\right), 2.91$ (br, $\left.1 \mathrm{H}, \mathrm{OAr}-\mathrm{CH}\left(\mathrm{CH}_{3}\right)_{2}\right), 2.60$ (br, $\left.2 \mathrm{H}, \mathrm{NbC}_{2} \mathrm{H}_{4}\right), 2.21\left(\mathrm{~s}, 3 \mathrm{H}, \mathrm{Ar}-\mathrm{CH}_{3}\right), 2.16\left(\mathrm{~s}, 3 \mathrm{H}, \mathrm{Ar}-\mathrm{CH}_{3}\right), 2.21-2.05$ (overlapped, $1 \mathrm{H}$, $\left.\mathrm{PCH}\left(\mathrm{CH}_{3}\right)_{2}\right), 1.69\left({ }^{3} J_{\mathrm{HH}}=6 \mathrm{~Hz},{ }^{2} J_{\mathrm{HP}}=14 \mathrm{~Hz}, \mathrm{PCH}\left(\mathrm{CH}_{3}\right)_{2}\right), 1.50\left(\mathrm{dd},{ }^{3} J_{\mathrm{HH}}=6 \mathrm{~Hz},{ }^{2} J_{\mathrm{HP}}\right.$ $\left.=14 \mathrm{~Hz}, \mathrm{PCH}\left(\mathrm{CH}_{3}\right)_{2}\right), 1.30-1.10$ (m, overlapped, $\left.18 \mathrm{H}, \mathrm{CH}\left(\mathrm{CH}_{3}\right)_{2}\right), 1.08-1.00(\mathrm{~m}$, overlapped, $\left.6 \mathrm{H}, \mathrm{CH}\left(\mathrm{CH}_{3}\right)_{2}\right), 0.98-0.85$ (m, overlapped, $\left.12 \mathrm{H}, \mathrm{CH}\left(\mathrm{CH}_{3}\right)_{2}\right), 0.65(\mathrm{dd}, 1 \mathrm{H}$, $\left.{ }^{3} J_{\mathrm{HH}}=6 \mathrm{~Hz},{ }^{2} J_{\mathrm{HP}}=15 \mathrm{~Hz}, \mathrm{PCH}\left(\mathrm{CH}_{3}\right)_{2}\right), 0.49\left(\mathrm{br} \mathrm{d},{ }^{3} J_{\mathrm{HH}}=6 \mathrm{~Hz}, 3 \mathrm{H}, \mathrm{NbCH}=\mathrm{CHCH}_{3}\right)$. ${ }^{13} \mathrm{C}\left\{{ }^{1} \mathrm{H}\right\}$ NMR $\left(126 \mathrm{MHz}\right.$, Benzene- $\left.d_{6}, 300 \mathrm{~K}\right): \delta 189.06\left(\mathrm{Nb}-\mathrm{CH}=\mathrm{CH}-\mathrm{CH}_{3}\right), 161.86$ (Ar), $156.99(\mathrm{Ar}), 140.83\left(\mathrm{~d}, J_{\mathrm{CP}}=20 \mathrm{~Hz}, \mathrm{Ar}\right), 134.08(\mathrm{Ar}), 132.90(\mathrm{Ar}), 132.26\left(\mathrm{~d}, J_{\mathrm{CP}}\right.$ $=15 \mathrm{~Hz}, \mathrm{Ar}), 131.37(\mathrm{Ar}), 126.70\left(\mathrm{~d}, J_{\mathrm{CP}}=4 \mathrm{~Hz}, \mathrm{Ar}\right), 124.11(\mathrm{Ar}), 123.87$ (Ar), 122.71 $\left(\mathrm{Nb}-\mathrm{CH}=\mathrm{CH}-\mathrm{CH}_{3}\right), 122.22\left(\mathrm{~d}, J_{\mathrm{CP}}=9 \mathrm{~Hz}, \mathrm{Ar}\right), 118.08\left(\mathrm{~d}, J_{\mathrm{CP}}=11 \mathrm{~Hz}, \mathrm{Ar}\right), 47.69(\mathrm{~d}$, $\left.{ }^{2} J_{\mathrm{CP}}=7 \mathrm{~Hz}, \mathrm{Nb}-\eta^{2}-\mathrm{C}_{2} \mathrm{H}_{4}\right), 47.62\left(\mathrm{~d},{ }^{2} J_{\mathrm{CP}}=8 \mathrm{~Hz}, \mathrm{Nb}-\eta^{2}-C_{2} \mathrm{H}_{4}\right), 34.46$ (pentane), 28.36 $\left(\mathrm{d},{ }^{1} J_{\mathrm{CP}}=17 \mathrm{~Hz}, \mathrm{PCH}\left(\mathrm{CH}_{3}\right)_{2}\right), 28.20\left(\mathrm{~d},{ }^{1} J_{\mathrm{CP}}=9 \mathrm{~Hz}, \mathrm{PCH}\left(\mathrm{CH}_{3}\right)_{2}\right), 25.77(\mathrm{OAr}-$ $\left.\mathrm{CH}\left(\mathrm{CH}_{3}\right)_{2}\right), 25.46\left(\mathrm{OAr}-\mathrm{CH}\left(\mathrm{CH}_{3}\right)_{2}\right), 25.28\left(\mathrm{OAr}-\mathrm{CH}\left(\mathrm{CH}_{3}\right)_{2}\right), 24.81\left(\mathrm{OAr}-\mathrm{CH}\left(\mathrm{CH}_{3}\right)_{2}\right)$, $24.11\left(\mathrm{OAr}-\mathrm{CH}\left(\mathrm{CH}_{3}\right)_{2}\right), 24.05\left(\mathrm{OAr}-\mathrm{CH}\left(\mathrm{CH}_{3}\right)_{2}\right), 23.75\left(\mathrm{Nb}-\mathrm{CH}=\mathrm{CH}-\mathrm{CH}_{3}\right), 22.75$ (pentane), 20.90 (br, $\left.\mathrm{PCH}\left(\mathrm{CH}_{3}\right)_{2}\right), 20.73\left(2 \mathrm{C}, \mathrm{ArCH}_{3}\right), 20.53\left(\mathrm{~d},{ }^{2} J_{\mathrm{CP}}=6 \mathrm{~Hz}\right.$, $\left.\operatorname{PCH}\left(\mathrm{CH}_{3}\right)_{2}\right), 20.03\left(\mathrm{~d},{ }^{2} J_{\mathrm{CP}}=6 \mathrm{~Hz}, \operatorname{PCH}\left(\mathrm{CH}_{3}\right)_{2}\right), 19.64$ (br (d, $\left.\mathrm{PCH}\left(\mathrm{CH}_{3}\right)_{2}\right), 19.25(\mathrm{~d}$, $\left.{ }^{2} J_{\mathrm{CP}}=6 \mathrm{~Hz}, \mathrm{PCH}\left(\mathrm{CH}_{3}\right)_{2}\right), 18.31\left(\mathrm{br}, \mathrm{PCH}\left(\mathrm{CH}_{3}\right)_{2}\right), 17.77$ (br, $\left.\mathrm{PCH}\left(\mathrm{CH}_{3}\right)_{2}\right), 17.25$ (d, 
$\left.{ }^{2} J_{\mathrm{CP}}=6 \mathrm{~Hz}, \mathrm{PCH}\left(\mathrm{CH}_{3}\right)_{2}\right), 14.32$ (pentane). ${ }^{31} \mathrm{P}\left\{{ }^{1} \mathrm{H}\right\}$ NMR (162 MHz, Benzene- $d_{6}, 300$ K) $\delta 45.15\left(\mathrm{br}, v_{1 / 2}=238 \mathrm{~Hz}, \mathrm{PNP}\right), 40.15\left(\mathrm{br}, v_{1 / 2}=297 \mathrm{~Hz}, \mathrm{PNP}\right)$. Anal. Calcd. for $\mathrm{C}_{43} \mathrm{H}_{66} \mathrm{NOP}_{2} \mathrm{Nb}: \mathrm{C}, 67.26 ; \mathrm{H}, 8.66 ; \mathrm{N}, 1.82$. Found: C, 67.11; H, 8.76; N, 1.80.

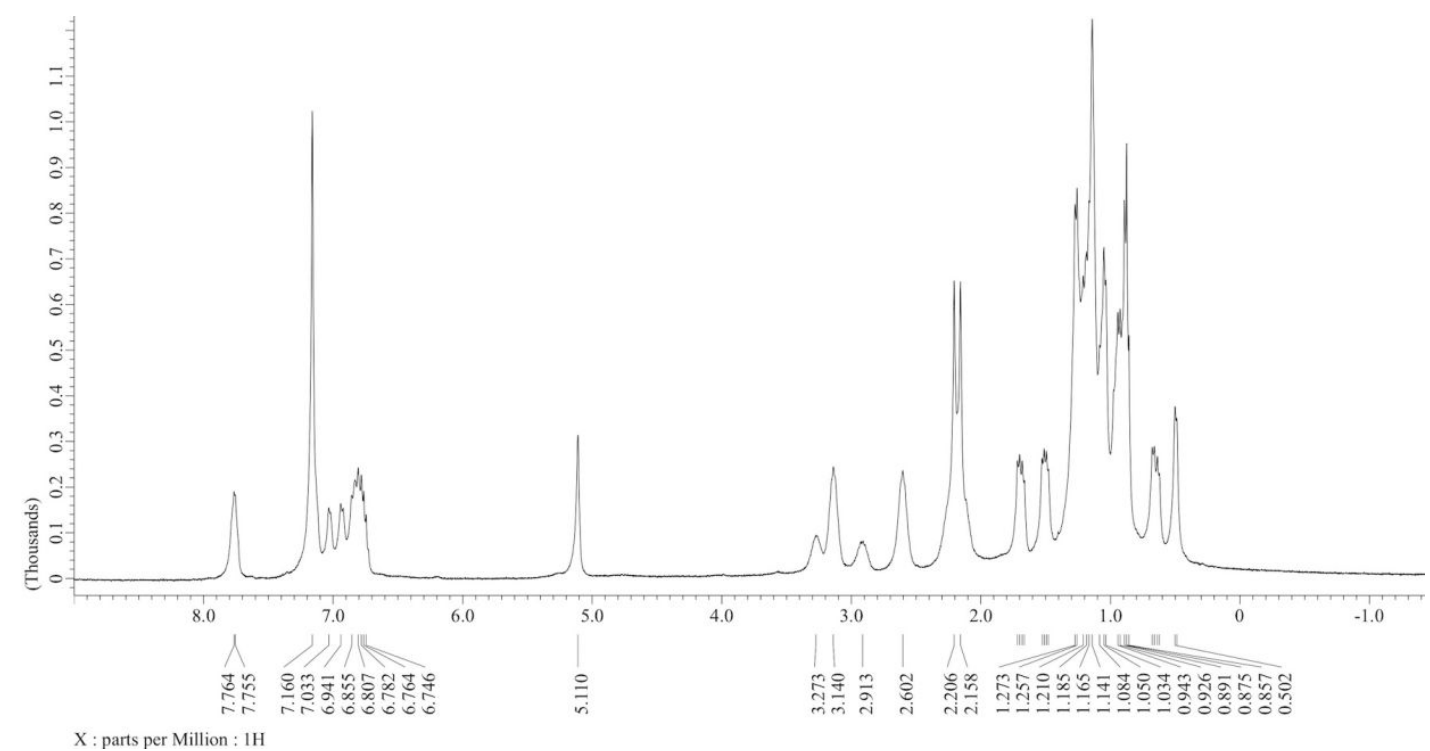

Figure S1. ${ }^{1} \mathrm{H}$ NMR spectrum of $2\left(400 \mathrm{MHz}\right.$, Benzene- $\left.d_{6}, 300 \mathrm{~K}\right)$.

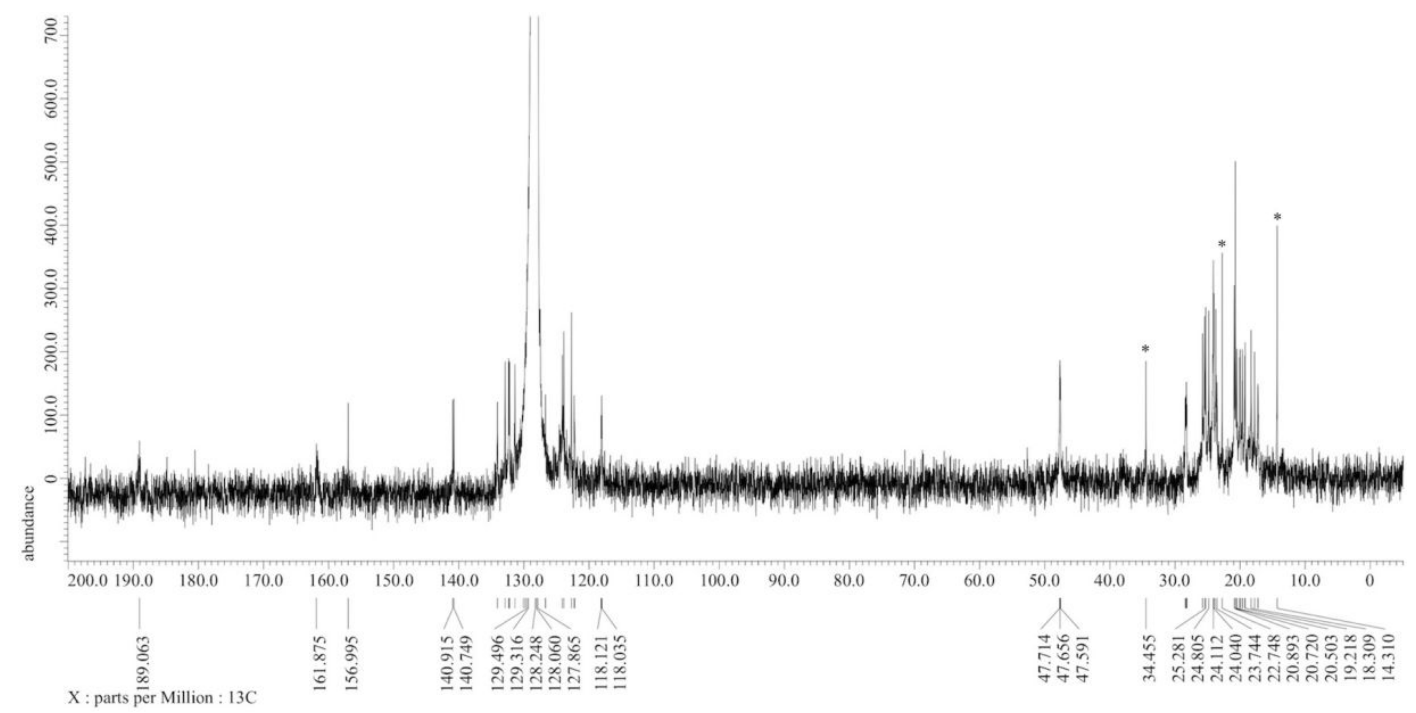

Figure S2. ${ }^{13} \mathrm{C}\left\{{ }^{1} \mathrm{H}\right\}$ NMR spectrum of $2\left(126 \mathrm{MHz}\right.$, Benzene- $\left.d_{6}, 300 \mathrm{~K}\right) .{ }^{*}$ : Residual solvent. 


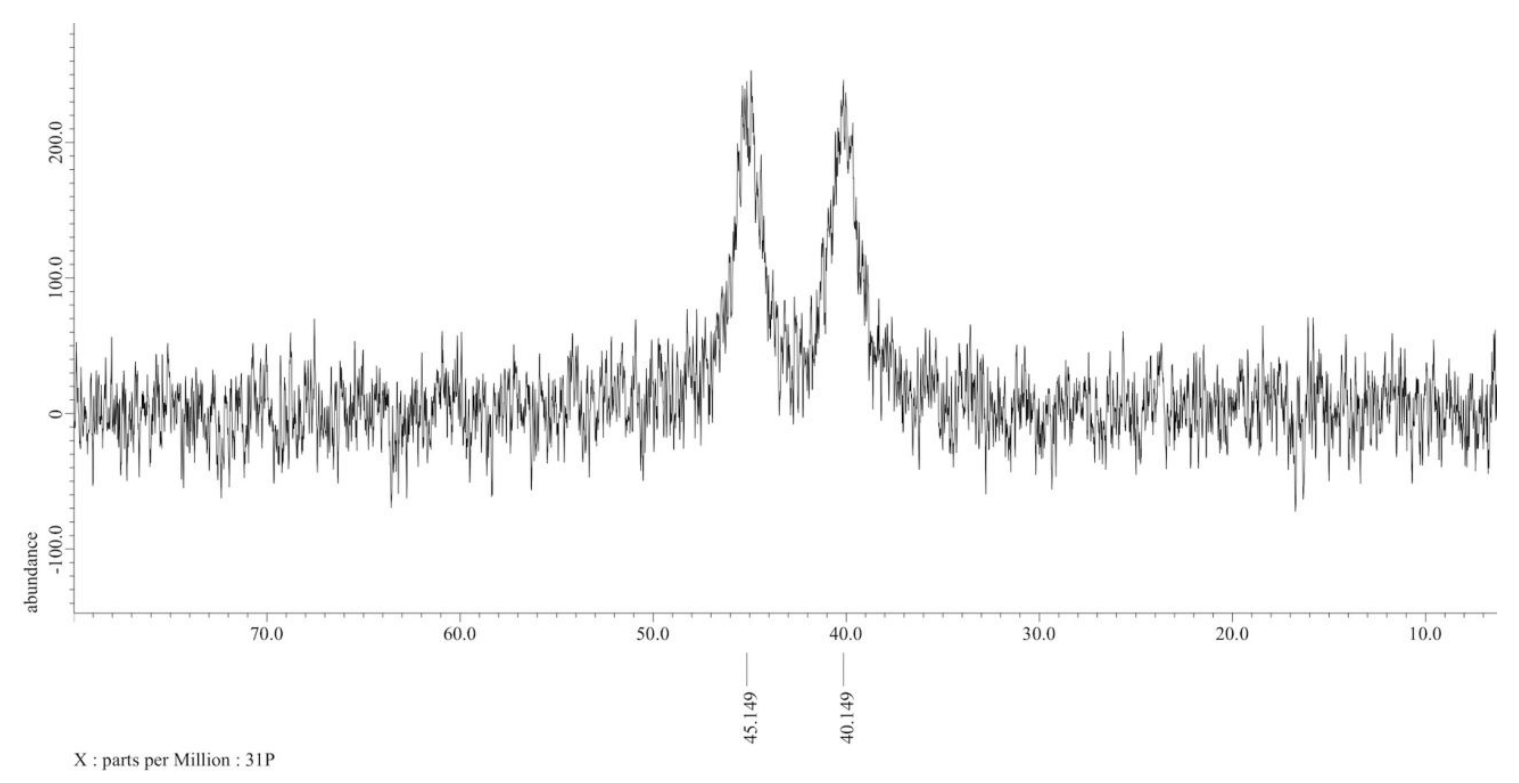

Figure S3. ${ }^{31} \mathrm{P}\left\{{ }^{1} \mathrm{H}\right\}$ NMR spectrum of $2\left(162 \mathrm{MHz}\right.$, Benzene- $\left.d_{6}, 300 \mathrm{~K}\right)$.

\section{Synthesis of $\left[(\mathrm{PNP}) \mathrm{Nb}\left(\eta^{3}-\mathrm{C}_{3} \mathrm{H}_{5}\right)(\mathrm{OAr})\right](3)$}

Under an $\mathrm{N}_{2}$ atmosphere, a reddish brown solution of $2(200 \mathrm{mg}, 445 \mu \mathrm{mol})$ in toluene $(10 \mathrm{~mL})$ was charged in a $100 \mathrm{~mL}$ Teflon valve tube. The reddish brown solution was heated at $80{ }^{\circ} \mathrm{C}$ and the color of the reaction mixture turned into slightly darker brown. After stirring for 18 hours at $80^{\circ} \mathrm{C}$, the reaction mixture was evaporated to dryness. The brown residue was dissolved in pentane $(5 \mathrm{~mL})$ and filtered through glass wool in a pipette. The brown filtrate was concentrated to ca. $1 \mathrm{~mL}$ and stored at $35{ }^{\circ} \mathrm{C}$ overnight to yield 3 as brown crystals (164 mg, $222 \mu \mathrm{mol}, 85 \%$ yield).

${ }^{1} \mathrm{H}$ NMR (400 MHz, Benzene- $\left.d_{6}, 300 \mathrm{~K}\right): \delta 7.26$ (br, 2H, Ar-CH), 7.10-6.85 (overlapped, 4H, Ar-CH), 6.90 (br t, 1H, Ar-CH), 6.81 (br, 2H, Ar-CH), 6.20 (br, 1H, $\mathrm{Nb}-\eta^{3}-\mathrm{CH}_{2} \mathrm{CHCH}_{2}$ ), 4.34 (br, $\left.1 \mathrm{H}, \mathrm{Nb}-\eta^{3}-\mathrm{CH}_{2} \mathrm{CHCH}_{2}\right), 3.59$ (br, $\left.1 \mathrm{H}, \mathrm{OAr}-\mathrm{CH}\left(\mathrm{CH}_{3}\right)_{2}\right)$, 2.69 (br, 1H, OAr-CH(CH$\left.)_{2}\right), 2.47$ (br, 4H, $\left.\mathrm{PCH}\left(\mathrm{CH}_{3}\right)_{2}\right), 2.17$ (s, 6H, Ar- $\left.\mathrm{CH}_{3}\right), 1.44$ (br, overlapped, 7H, $\mathrm{PCH}\left(\mathrm{CH}_{3}\right)_{2}$ and $\left.\mathrm{Nb}-\eta^{3}-\mathrm{CH}_{2} \mathrm{CHCH}_{2}\right), 1.26\left(\right.$ br, $12 \mathrm{H}, \mathrm{PCH}\left(\mathrm{CH}_{3}\right)_{2}$ and $\left.\mathrm{OAr}-\mathrm{CH}\left(\mathrm{CH}_{3}\right)_{2}\right), 1.07$ (br, overlapped, 7H, OAr- $\mathrm{CH}\left(\mathrm{CH}_{3}\right)_{2}$ and $\mathrm{Nb}-\eta^{3}-$ $\mathrm{CH}_{2} \mathrm{CHCH}_{2}$ ), 0.88 (br, 6H, $\left.\mathrm{PCH}\left(\mathrm{CH}_{3}\right)_{2}\right), 0.69$ (br, overlapped, $\left.6 \mathrm{H}, \mathrm{PCH}\left(\mathrm{CH}_{3}\right)_{2}\right),-0.28$ (br, $\left.1 \mathrm{H}, \mathrm{Nb}-\eta^{3}-\mathrm{CH}_{2} \mathrm{CHCH}_{2}\right) \cdot{ }^{13} \mathrm{C}\left\{{ }^{1} \mathrm{H}\right\} \mathrm{NMR}\left(101 \mathrm{MHz}\right.$, Benzene- $\left.d_{6}, 300 \mathrm{~K}\right): \delta 156.70$ (Ar), 139.60 (Ar), 131.41 (Ar), 127.00 (Ar), 123.63 (Ar), 123.39 (Ar), 122.23 (Ar), 
$81.08\left(\mathrm{Nb}-\eta^{3}-\mathrm{CH}_{2} \mathrm{CHCH}_{2}\right), 25.58\left(\mathrm{OAr}-\mathrm{CH}\left(\mathrm{CH}_{3}\right)_{2}\right.$ or $\left.\mathrm{OAr}-\mathrm{CH}\left(\mathrm{CH}_{3}\right)_{2}\right), 25.49(\mathrm{OAr}-$ $\mathrm{CH}\left(\mathrm{CH}_{3}\right)_{2} \quad$ or $\left.\quad \mathrm{OAr}-\mathrm{CH}\left(\mathrm{CH}_{3}\right)_{2}\right), \quad 24.91 \quad\left(\mathrm{PCH}\left(\mathrm{CH}_{3}\right)_{2}\right), \quad 20.76 \quad\left(\mathrm{ArCH}_{3}\right), \quad 19.18$ $\left(\mathrm{PCH}\left(\mathrm{CH}_{3}\right)_{2}\right), 19.09\left(\mathrm{PCH}\left(\mathrm{CH}_{3}\right)_{2}\right), 18.75\left(\mathrm{PCH}\left(\mathrm{CH}_{3}\right)_{2}\right)$. Some of the ${ }^{13} \mathrm{C}$ NMR signals have been assigned by ${ }^{1} \mathrm{H}-{ }^{13} \mathrm{C}$ HSQC measurement. Fluxional behavior in solution prevented us from locating some ${ }^{13} \mathrm{C}$ NMR signals in the PNP and OAr ligands. ${ }^{31} \mathrm{P}\left\{{ }^{1} \mathrm{H}\right\}$ NMR (162 MHz, Benzene- $\left.d_{6}, 300 \mathrm{~K}\right) \delta 50.69$ (br, $\left.v_{1 / 2}=364 \mathrm{~Hz}, \mathrm{PNP}\right), 48.02$ (br, $v_{1 / 2}$ $=296 \mathrm{~Hz}, \mathrm{PNP})$. Anal. Calcd. for $\mathrm{C}_{41} \mathrm{H}_{62} \mathrm{NOP}_{2} \mathrm{Nb}: \mathrm{C}, 66.56 ; \mathrm{H}, 8.45 ; \mathrm{N}, 1.89$. Found: C, 66.72; H, 8.57; N, 1.81 .

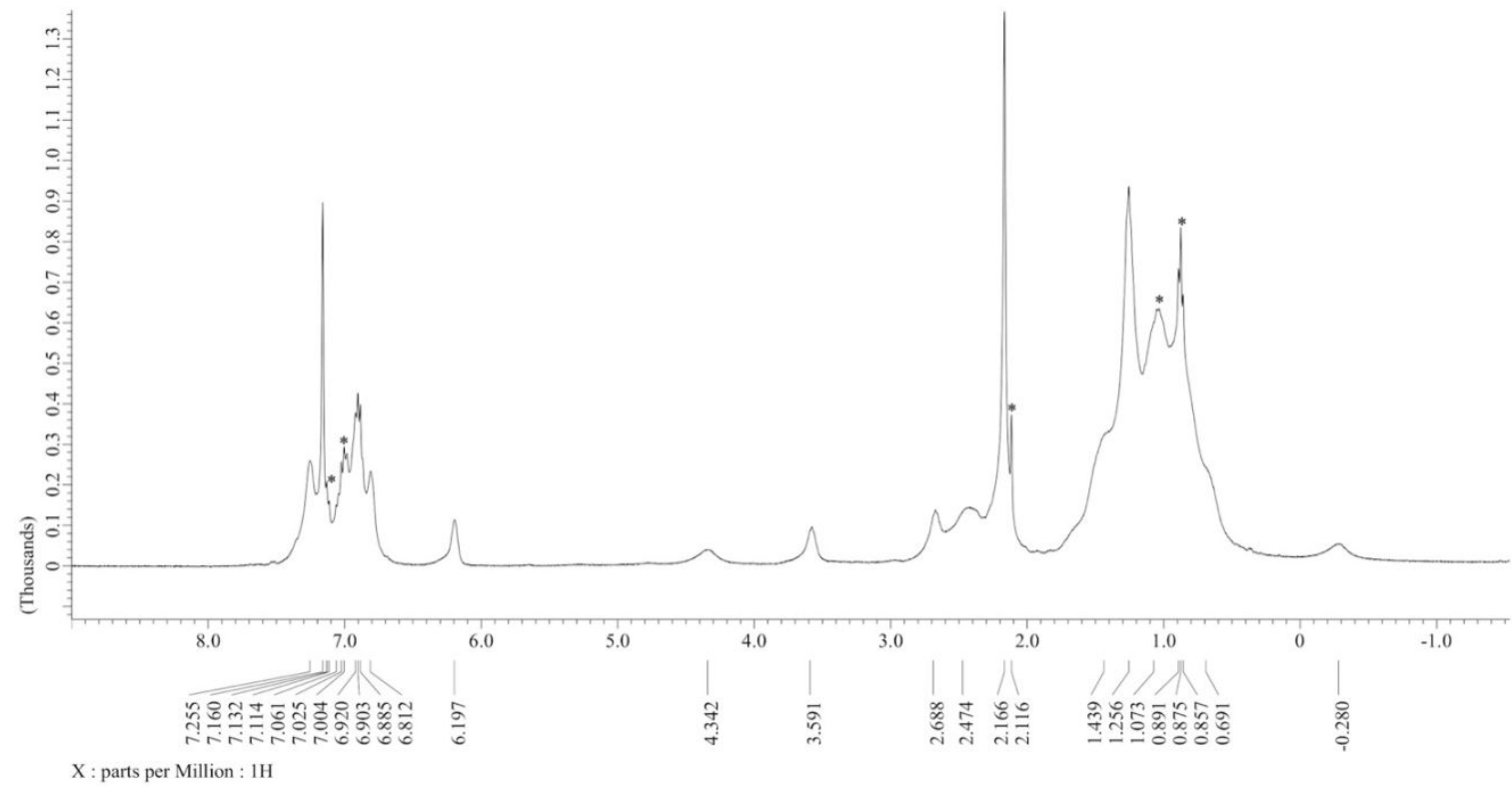

Figure S4. ${ }^{1} \mathrm{H}$ NMR spectrum of 3 (400 MHz, Benzene- $\left.d_{6}, 300 \mathrm{~K}\right)$. *: Residual solvents. 


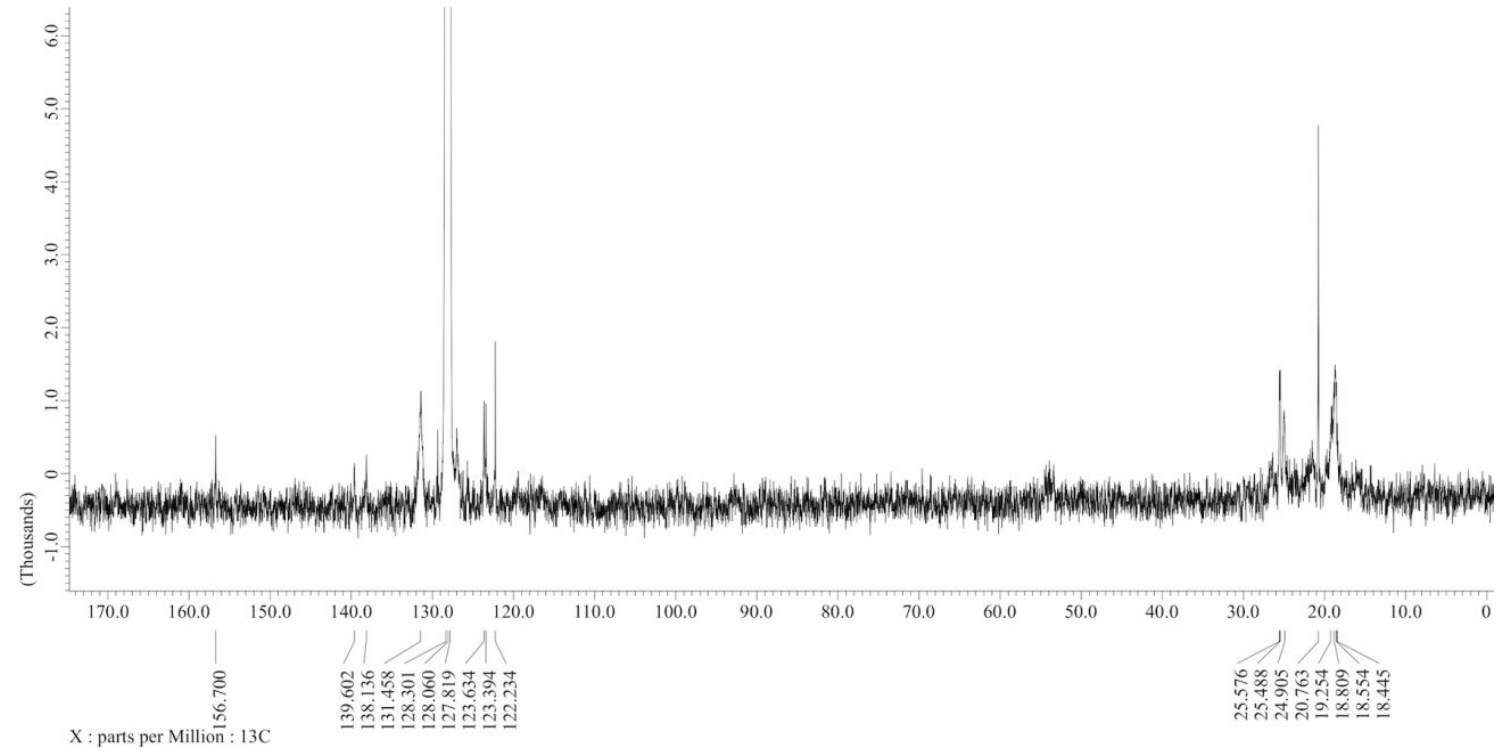

Figure S5. ${ }^{13} \mathrm{C}\left\{{ }^{1} \mathrm{H}\right\}$ NMR spectrum of $3\left(101 \mathrm{MHz}\right.$, Benzene- $\left.d_{6}, 300 \mathrm{~K}\right)$.

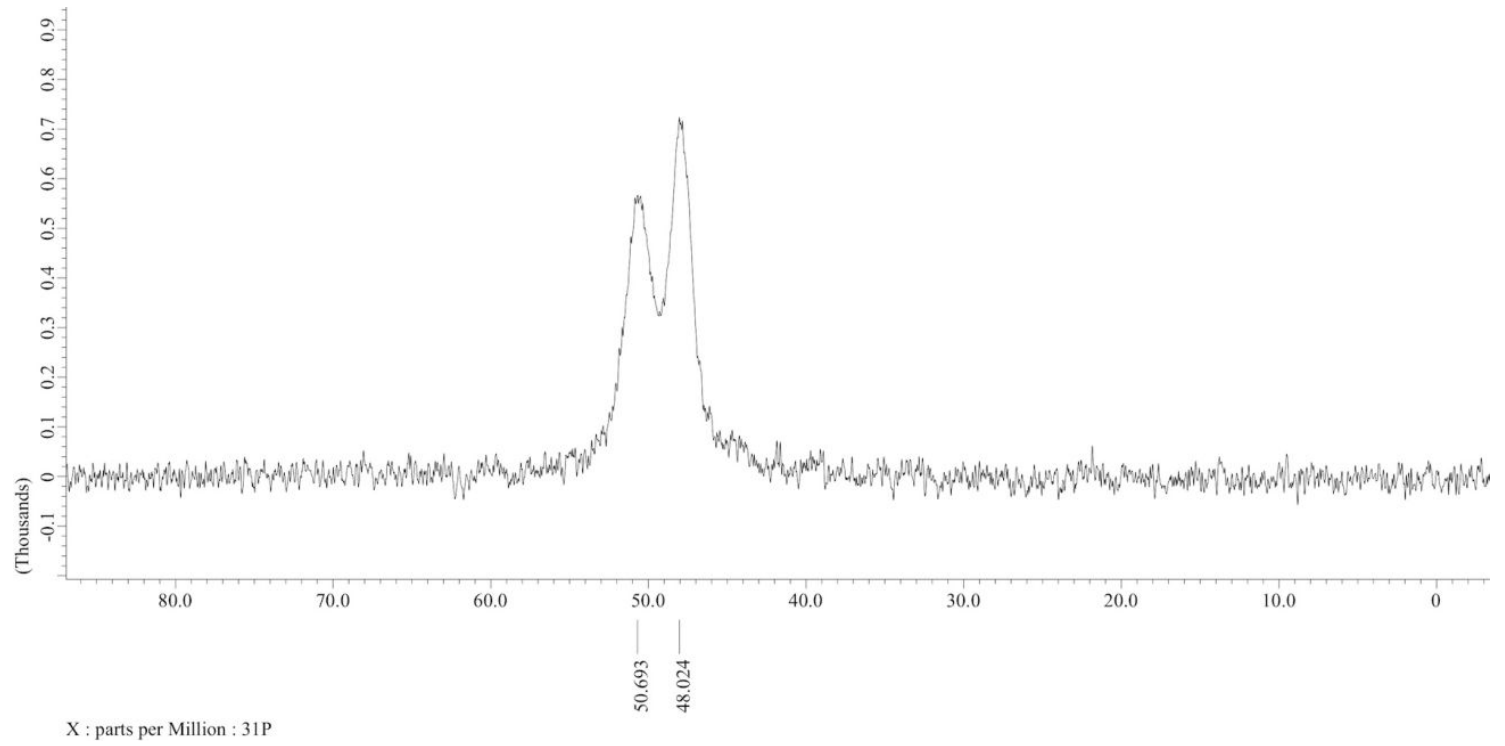

Figure S6. ${ }^{31} \mathrm{P}\left\{{ }^{1} \mathrm{H}\right\}$ NMR spectrum of $3\left(162 \mathrm{MHz}\right.$, Benzene- $\left.d_{6}, 300 \mathrm{~K}\right)$. 


\section{NMR Monitoring Reaction of 1 with $\mathrm{C}_{2} \mathrm{H}_{4}$}

A green solution of $1(16.1 \mathrm{mg}, 28.7 \mu \mathrm{mol})$ in benzene- $d_{6}(\mathrm{ca} .0 .5 \mathrm{~mL})$ was charged in a J-Young valve NMR tube. The solution was frozen by liquid $\mathrm{N}_{2}$ and the headspace was evacuated. The solution was thawed at room temperature and an atmosphere of $\mathrm{C}_{2} \mathrm{H}_{4}(1 \mathrm{~atm})$ was introduced into the NMR tube. The reaction mixture gradually changed in color from green to reddish brown over 4 hours at room temperature. ${ }^{1} \mathrm{H}$ NMR and ${ }^{31} \mathrm{P}\left\{{ }^{1} \mathrm{H}\right\}$ NMR spectra were measured after 30 minutes and 4 hours. After 30 minutes at room temperature, the ${ }^{31} \mathrm{P}\left\{{ }^{1} \mathrm{H}\right\}$ NMR spectrum showed a mixture of three different $[(\mathrm{PNP}) \mathrm{Nb}]$ species (Figure S8). Two of them were confirmed as $[(\mathrm{PNP}) \mathrm{Nb}(\mathrm{CH}) \mathrm{OAr}](\mathbf{1})$ and $\left[(\mathrm{PNP}) \mathrm{Nb}\left(\mathrm{CH}=\mathrm{CHCH}_{3}\right)\left(\eta^{2}-\mathrm{C}_{2} \mathrm{H}_{4}\right)(\mathrm{OAr})\right](\mathbf{2})$. The other [(PNP)Nb] species at $48.18 \mathrm{ppm}$ and $50.78 \mathrm{ppm}$ as broad doublets could be a metallacyclobutene intermediate [(PNP)Nb$\left.\left(\mathrm{CHCH}_{2} \mathrm{CH}_{2}\right)(\mathrm{OAr})\right]$ (A). The ${ }^{1} \mathrm{H}$ NMR spectrum showed a doublet $(J=16 \mathrm{~Hz})$ at $8.10 \mathrm{ppm}$ as well as the methylidyne signal $[\mathrm{NbCH}]$ at $6.01 \mathrm{ppm}(\mathbf{1})$ and the vinylic signal $\left[\mathrm{Nb}-\mathrm{CH}=\mathrm{CH}-\mathrm{CH}_{3}\right](2)$ at $5.11 \mathrm{ppm}$ (Figure S7). After 4 hours at room temperature, the ${ }^{31} \mathrm{P}\left\{{ }^{1} \mathrm{H}\right\}$ NMR spectrum (Figure S10) showed almost a single [(PNP)Nb] compound, which was confirmed as $\left[(\mathrm{PNP}) \mathrm{Nb}\left(\mathrm{CH}=\mathrm{CHCH}_{3}\right)\left(\eta^{2}-\mathrm{C}_{2} \mathrm{H}_{4}\right)(\mathrm{OAr})\right](2)$. In the ${ }^{1} \mathrm{H}$ NMR spectrum after 4 hours (Figure S9), the double peak at $8.10 \mathrm{ppm}$ disappeared and almost quantitative formation of 2 was observed. 


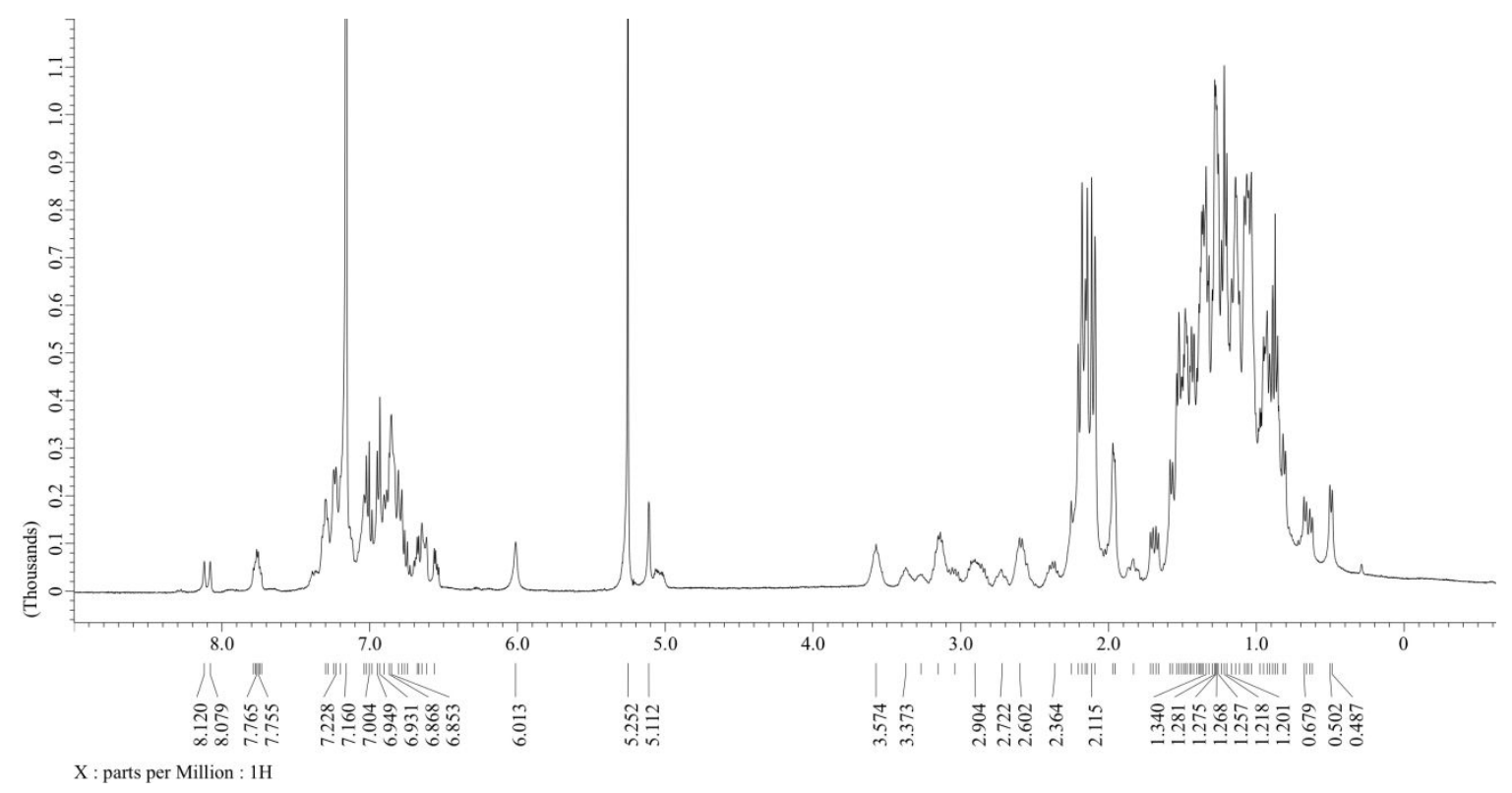

Figure S7. ${ }^{1} \mathrm{H}$ NMR spectrum of 1 with $\mathrm{C}_{2} \mathrm{H}_{4}$ after 30 minutes (400 MHz, Benzene- $d_{6}$, $300 \mathrm{~K})$.

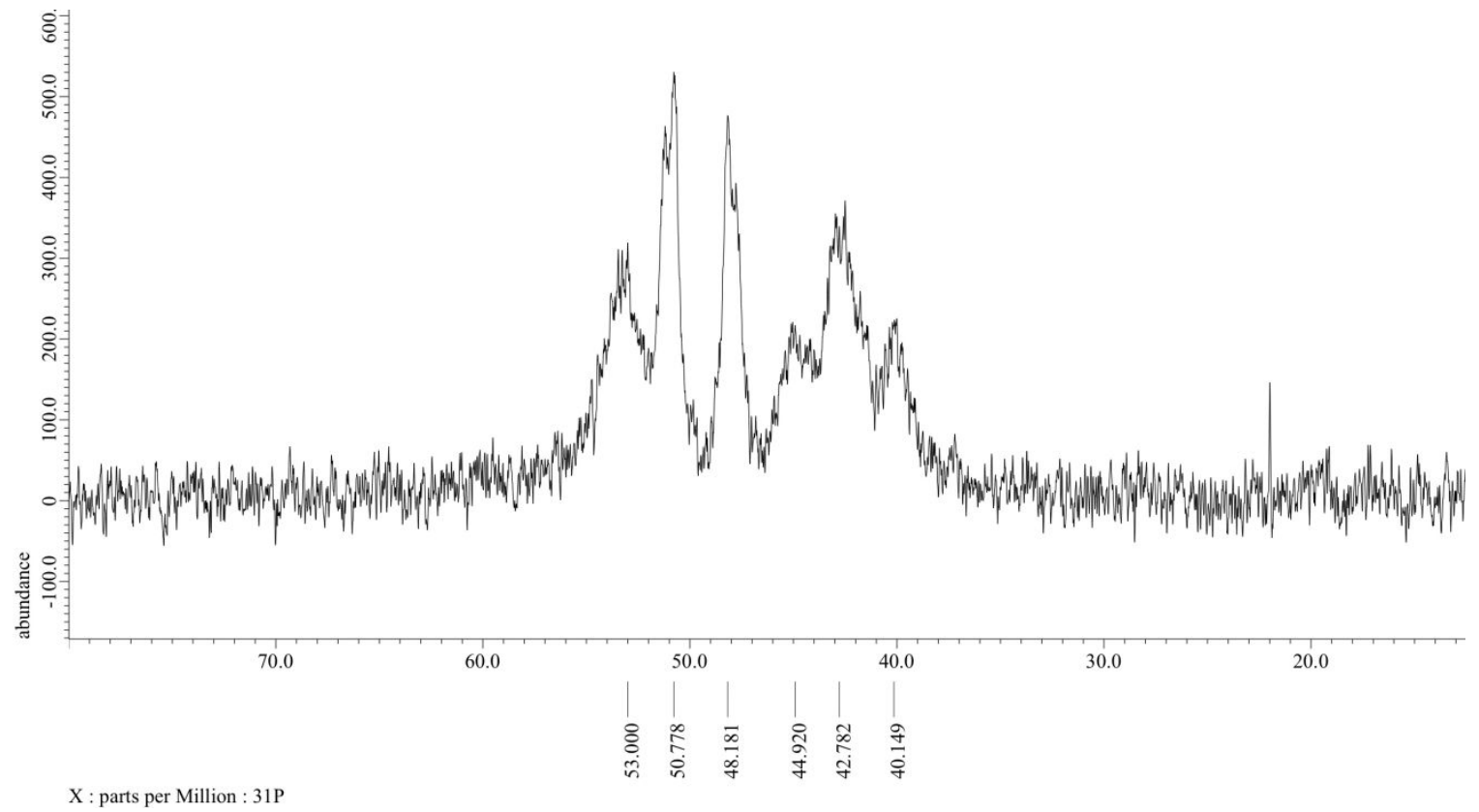

Figure S8. ${ }^{31} \mathrm{P}\left\{{ }^{1} \mathrm{H}\right\}$ NMR spectrum of 1 with $\mathrm{C}_{2} \mathrm{H}_{4}$ after 30 minutes $(162 \mathrm{MHz}$, Benzene- $d_{6}, 300 \mathrm{~K}$ ). 


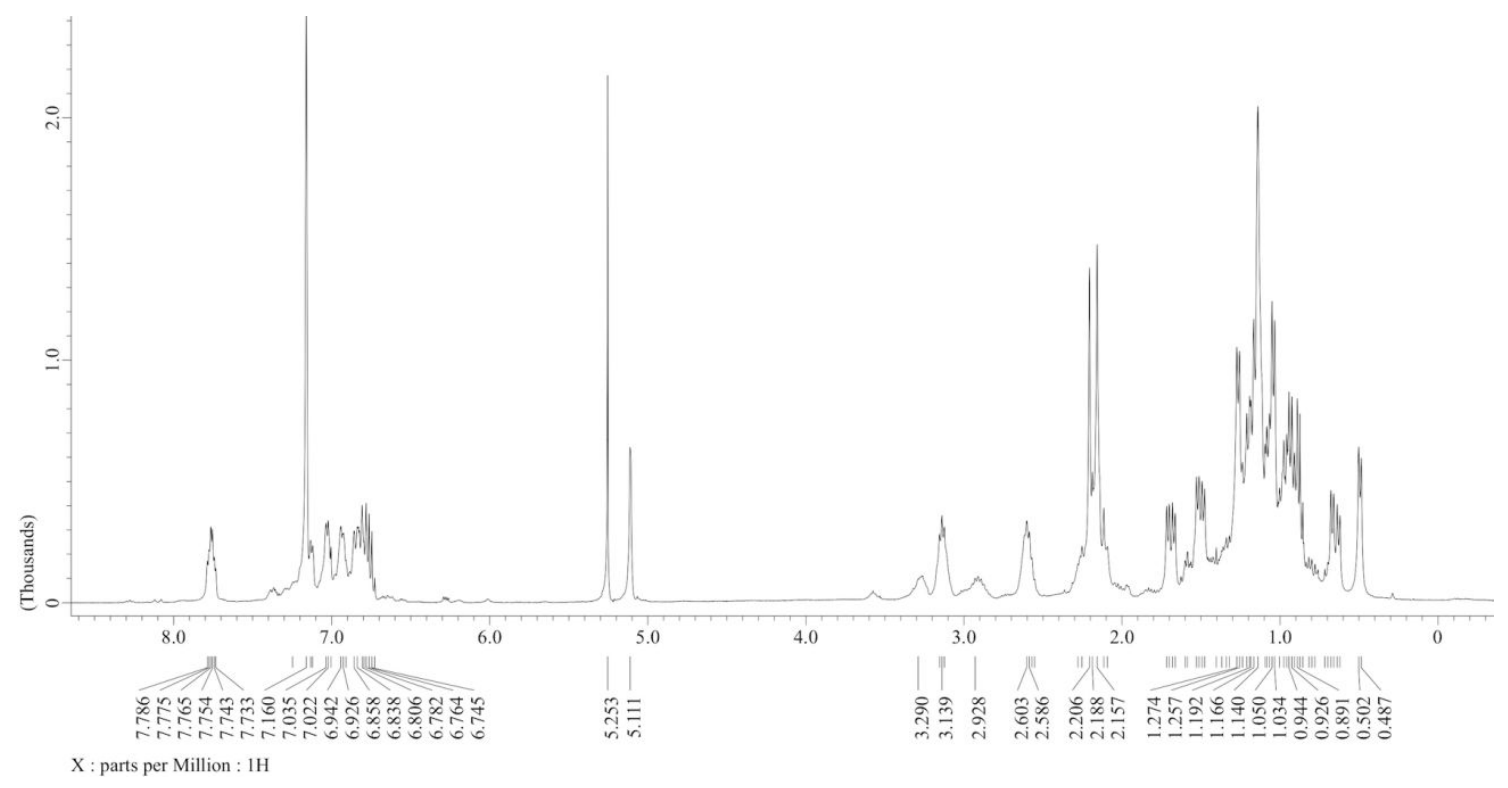

Figure S9. ${ }^{1} \mathrm{H}$ NMR spectrum of 1 with $\mathrm{C}_{2} \mathrm{H}_{4}$ after 4 hours $\left(400 \mathrm{MHz}\right.$, Benzene- $d_{6}, 300$ K).

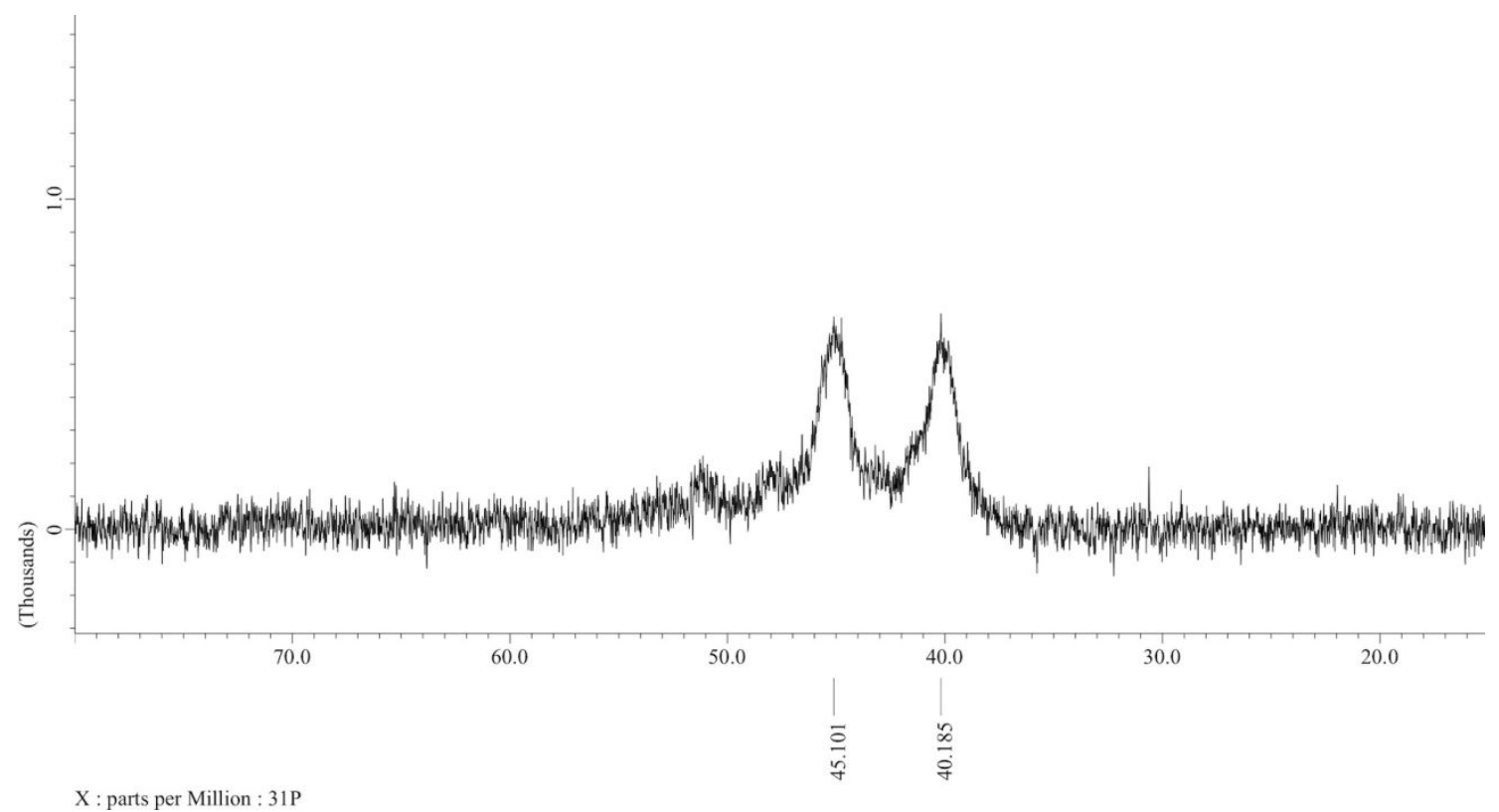

Figure S10. ${ }^{31} \mathrm{P}\left\{{ }^{1} \mathrm{H}\right\}$ NMR spectrum of 1 with $\mathrm{C}_{2} \mathrm{H}_{4}$ after 4 hours $(162 \mathrm{MHz}$, Benzene$\left.d_{6}, 300 \mathrm{~K}\right)$. 


\section{NMR Monitoring Reaction of ${ }^{1-13} \mathrm{C}$ with $\mathrm{C}_{2} \mathrm{H}_{4}$}

A green solution of $\mathbf{1 -}^{13} \mathbf{C} \cdot 0.5\left(\mathrm{FeCp}_{2}\right)(16.1 \mathrm{mg}, 28.7 \mu \mathrm{mol})$ in benzene- $d_{6}$ (ca. 0.5 $\mathrm{mL}$ ) was charged in a J-Young valve NMR tube. The solution was frozen by liquid $\mathrm{N}_{2}$ and the headspace was evacuated. The solution was thawed at room temperature and an atmosphere of $\mathrm{C}_{2} \mathrm{H}_{4}(1 \mathrm{~atm})$ was introduced into the NMR tube. The reaction mixture gradually changed in color from green to reddish brown over 4 hours at room temperature. ${ }^{1} \mathrm{H}$ NMR and ${ }^{31} \mathrm{P}\left\{{ }^{1} \mathrm{H}\right\}$ NMR spectra were measured after 30 minutes. In the ${ }^{1} \mathrm{H}$ NMR spectrum (Figure S11), a doublet of doublets was observed at $8.10 \mathrm{ppm}$ with a ${ }^{1} \mathrm{H}-{ }^{13} \mathrm{C}$ coupling ${ }^{1} J_{\mathrm{CH}}=108 \mathrm{~Hz}$ corresponding to $\mathbf{A}-{ }^{13} \mathbf{C}$ as well as a doublet of unreacted $\left[\mathrm{Nb}^{13} \mathrm{CH}\right]\left(\mathbf{1}-{ }^{13} \mathbf{C}\right)$ at $6.01 \mathrm{ppm}\left({ }^{1} J_{\mathrm{CH}}=134 \mathrm{~Hz}\right)$. The ${ }^{31} \mathrm{P}\left\{{ }^{1} \mathrm{H}\right\}$ NMR spectrum showed much more broaden signals of $[(\mathrm{PNP}) \mathrm{Nb}]$ species due to coupling of ${ }^{13} \mathrm{C}$ and ${ }^{31} \mathrm{P}$ nuclei as well as coupling with ${ }^{97} \mathrm{Nb}$ nuclear. ${ }^{13} \mathrm{C}\left\{{ }^{1} \mathrm{H}\right\}$ NMR and ${ }^{1} \mathrm{H}-{ }^{13} \mathrm{C}$ HSQC NMR spectra were measured quickly with a short period of measurement time. The ${ }^{13} \mathrm{C}\left\{{ }^{1} \mathrm{H}\right\}$ NMR spectrum (Figure S12) showed two enriched ${ }^{13} \mathrm{C}$ NMR signals at 176.42 $\operatorname{ppm}\left(\mathbf{A}-{ }^{13} \mathbf{C}\right)$ with ${ }^{2} J_{\mathrm{CP}}=15 \mathrm{~Hz}$ and $189.06 \mathrm{ppm}\left(\mathbf{2 -}{ }^{\mathbf{1 3}} \mathbf{C}\right)$, but the methylidyne region for $\left[\mathrm{Nb}^{13} \mathrm{CH}\right]\left(\mathbf{1 - 1 3}^{13} \mathrm{C}\right)$ was not measured. The ${ }^{1} \mathrm{H}-{ }^{13} \mathrm{C}$ HSQC NMR spectra (Figure S13 and S14) showed strong cross peaks $\delta{ }^{1} \mathrm{H}^{13} \mathrm{C}$ at $\delta 8.10-176.42\left(\mathbf{A}^{-13} \mathbf{C}\right)$ and $\delta 5.11-189.06$ for $\left(2-{ }^{13} \mathbf{C}\right)$. After 4 hours at room temperature, the ${ }^{13} \mathrm{C}\left\{{ }^{1} \mathrm{H}\right\}$ NMR spectrum (Figure S16) showed only one enriched ${ }^{13} \mathrm{C}$ NMR signal of $\mathbf{2 -}^{13} \mathbf{C}$ at $189.06 \mathrm{ppm}$. The NMR tube was evacuated to remove excessive $\mathrm{C}_{2} \mathrm{H}_{4}$ and charged with an $\mathrm{N}_{2}$ atmosphere. The ${ }^{1} \mathrm{H}$ NMR spectrum (Figure S15, bottom) showed one broad doublet and one broad doublet of doublets at $5.11 \mathrm{ppm}$, where only one broad signal appears for non-labeled 2. These two sets of broad signals are corresponding to $\alpha-\mathrm{CH}$ (br dd, ${ }^{1} J_{\mathrm{CH}}=118 \mathrm{~Hz}$, $\left.{ }^{3} J_{\mathrm{HH}}=16 \mathrm{~Hz}\right)$ and $\beta-\mathrm{CH}\left(\right.$ br $\left.\mathrm{d},{ }^{3} J_{\mathrm{HH}}=16 \mathrm{~Hz}\right)$ of $\left[(\mathrm{PNP}) \mathrm{Nb}\left({ }^{13} \mathrm{CH}=\mathrm{CHCH}_{3}\right)\left(\eta^{2}-\right.\right.$ $\left.\left.\mathrm{C}_{2} \mathrm{H}_{4}\right)(\mathrm{OAr})\right]\left(2-{ }^{13} \mathrm{C}\right)$. 


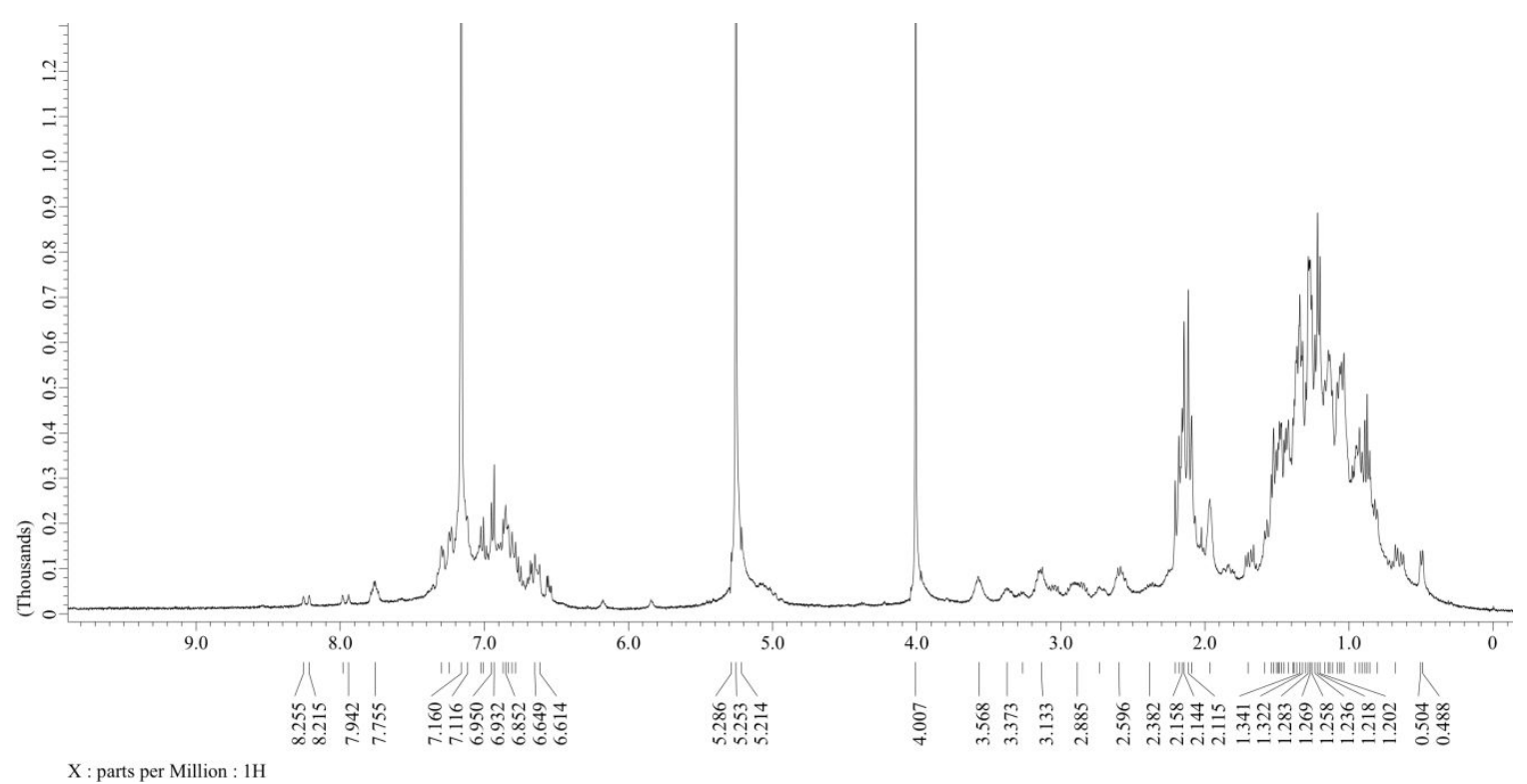

Figure S11. ${ }^{1} \mathrm{H}$ NMR spectrum of $\mathbf{1 -}^{13} \mathbf{C}$ with $\mathrm{C}_{2} \mathrm{H}_{4}$ after 30 minutes $(400 \mathrm{MHz}$, in Benzene- $d_{6}$, at $\left.300 \mathrm{~K}\right)$.

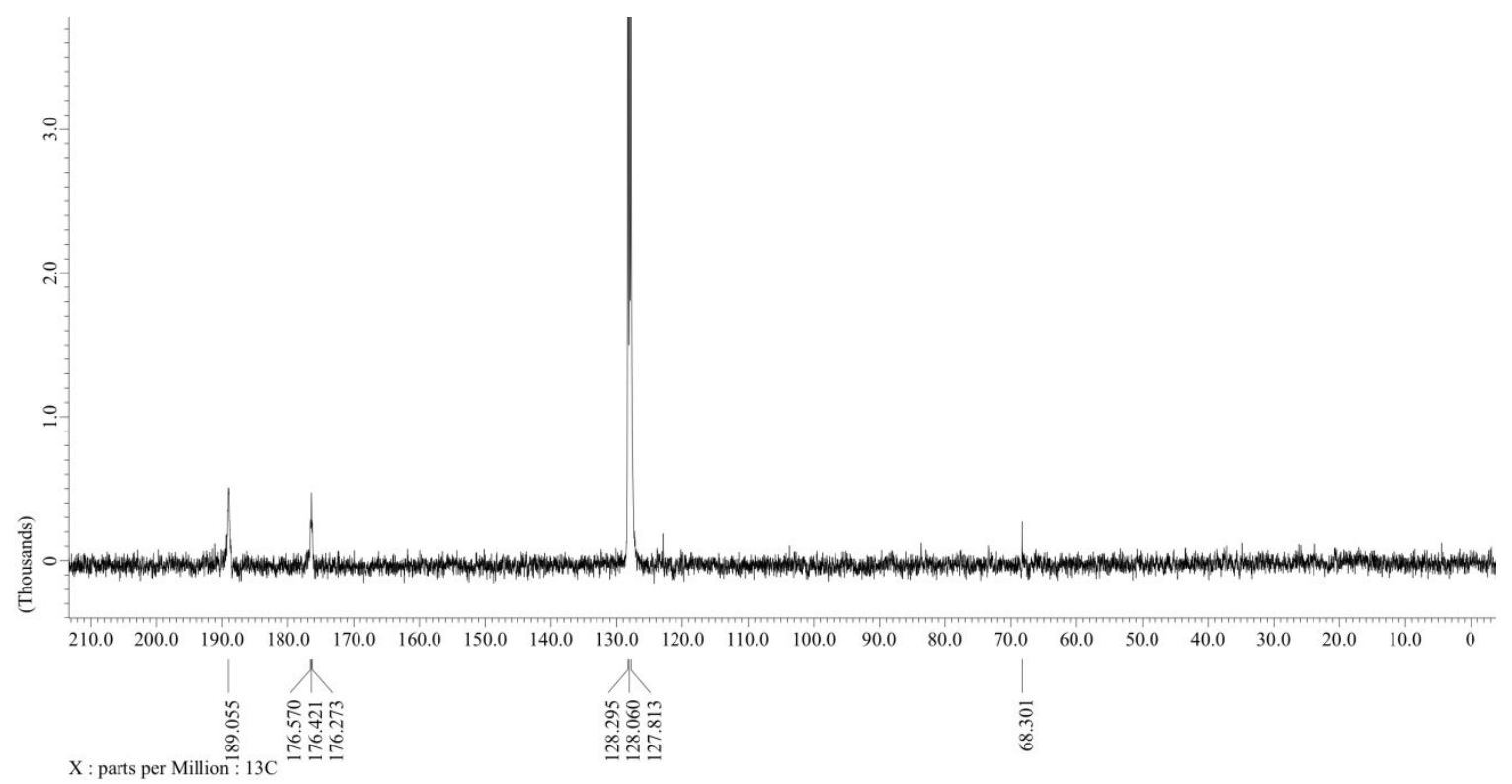

Figure S12. ${ }^{13} \mathrm{C}\left\{{ }^{1} \mathrm{H}\right\}$ NMR spectrum of $1-{ }^{13} \mathbf{C}$ with $\mathrm{C}_{2} \mathrm{H}_{4}$ after 40 minutes $(101 \mathrm{MHz}$, Benzene- $\left.d_{6}, 300 \mathrm{~K}\right)$. 


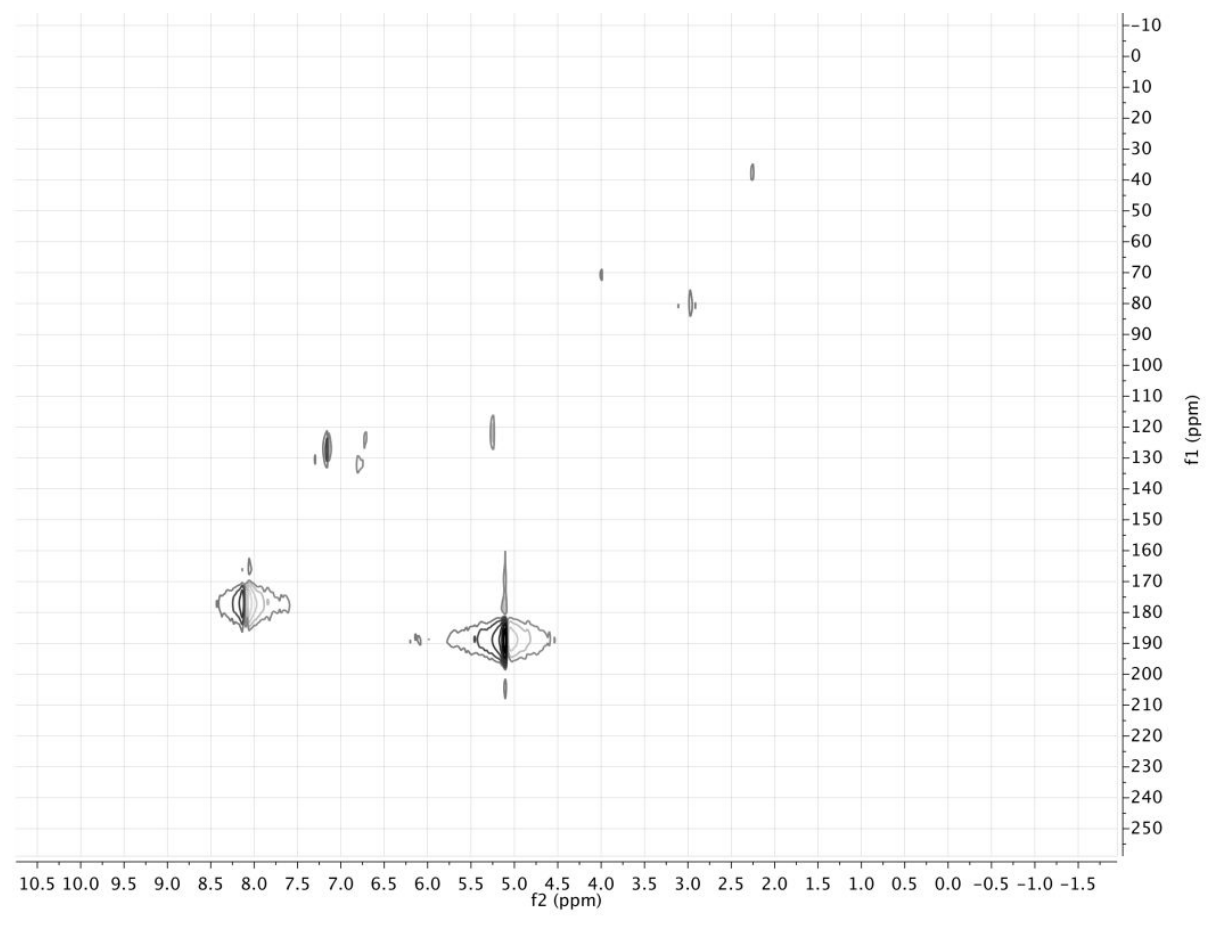

Figure S13. Decoupled ${ }^{1} \mathrm{H}-{ }^{13} \mathrm{C}$ HSQC NMR spectrum of ${ }^{1-13} \mathrm{C}$ with $\mathrm{C}_{2} \mathrm{H}_{4}$ after 60 minutes $\left(101 \mathrm{MHz}\right.$, Benzene- $\left.d_{6}, 300 \mathrm{~K}\right)$.

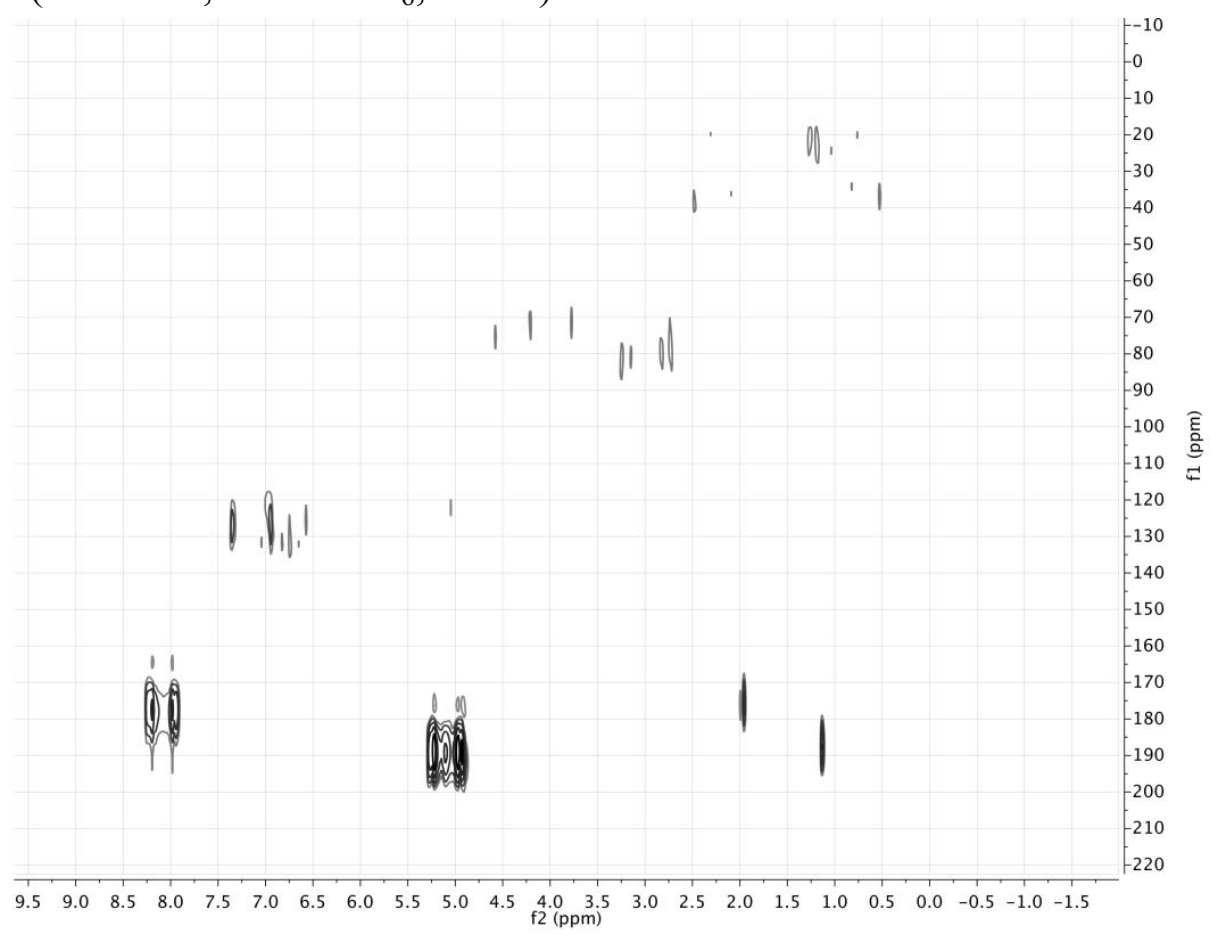

Figure S14. Non-decoupled ${ }^{1} \mathrm{H}-{ }^{13} \mathrm{C}$ HSQC NMR spectrum of $1-{ }^{13} \mathbf{C}$ with $\mathrm{C}_{2} \mathrm{H}_{4}$ after 70 minutes $\left(101 \mathrm{MHz}\right.$, Benzene- $\left.d_{6}, 300 \mathrm{~K}\right)$. 

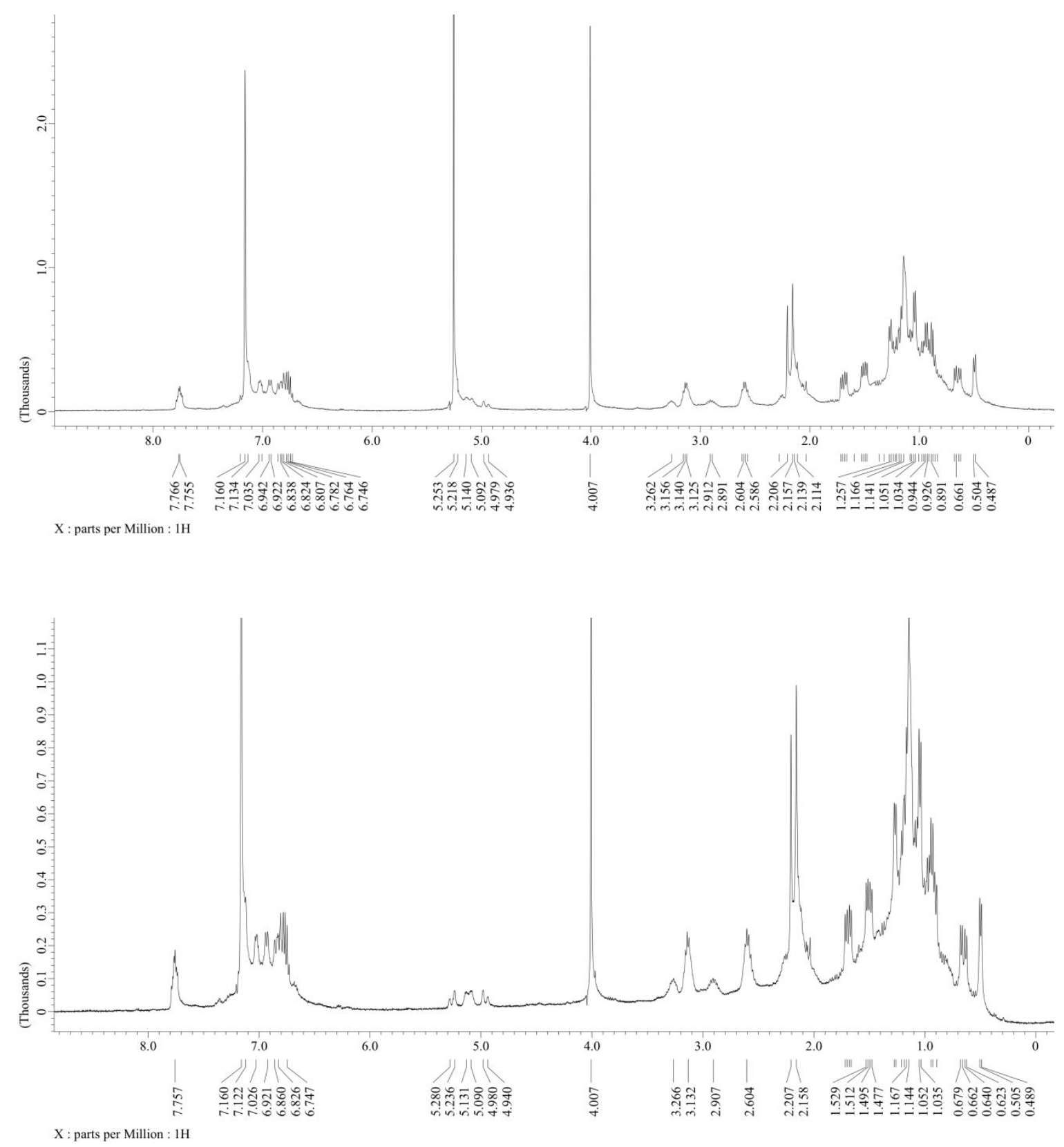

Figure S15. ${ }^{1} \mathrm{H}$ NMR spectrum of $\mathbf{1 - 1}^{13} \mathbf{C}$ with $\mathrm{C}_{2} \mathrm{H}_{4}$ after 4 hours $(400 \mathrm{MHz}$, in Benzene$d_{6}$, at $300 \mathrm{~K}$ ). Top: under $\mathrm{C}_{2} \mathrm{H}_{4}$. Bottom: under $\mathrm{N}_{2}$. 


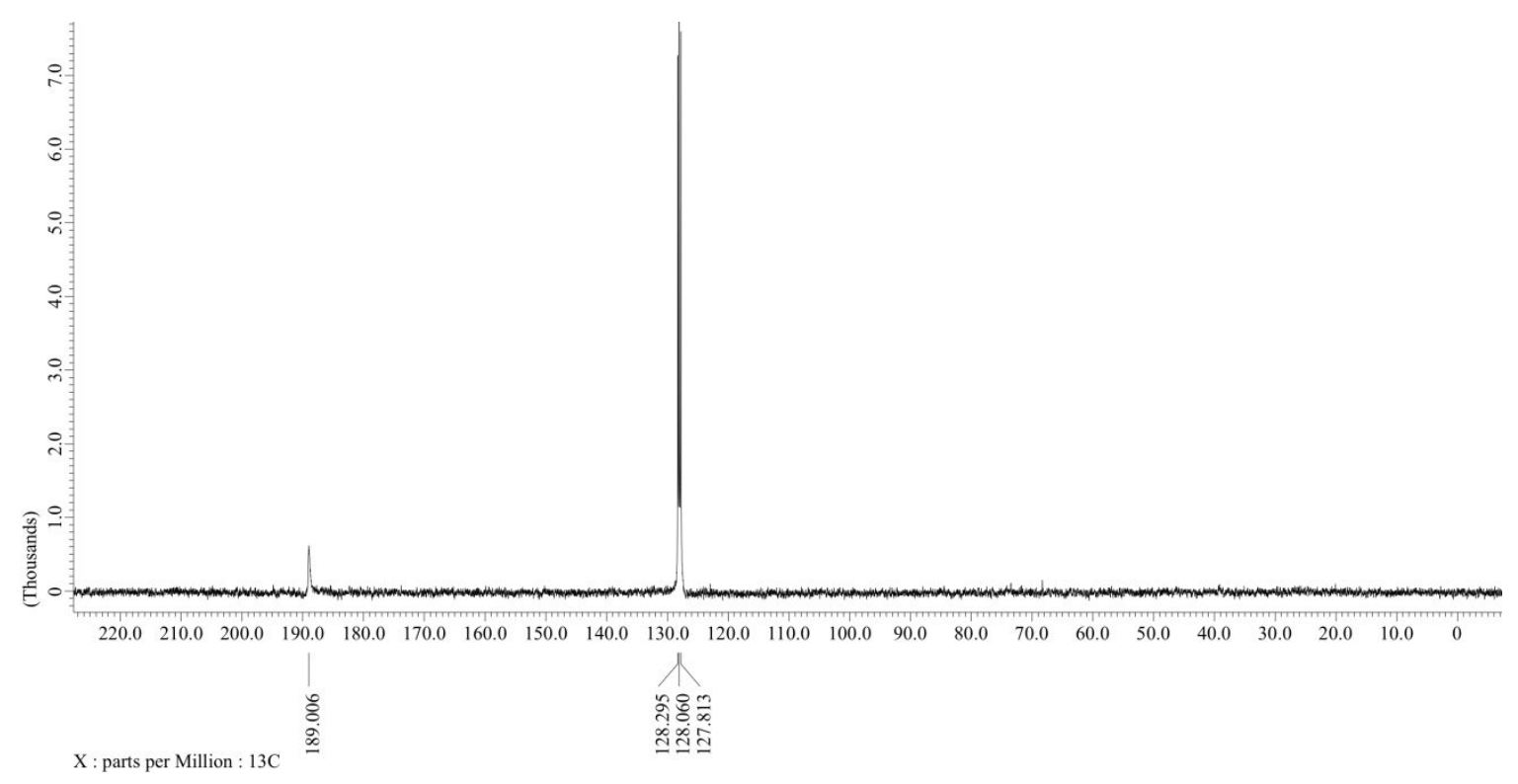

Figure S16. ${ }^{13} \mathrm{C}\left\{{ }^{1} \mathrm{H}\right\}$ NMR spectrum of $\mathbf{1 -}^{13} \mathbf{C}$ with $\mathrm{C}_{2} \mathrm{H}_{4}$ after 4 hours $(101 \mathrm{MHz}$, Benzene- $\left.d_{6}, 300 \mathrm{~K}\right)$.

\section{NMR Monitoring Reaction of $2-{ }^{13} \mathrm{C}$ under $\mathrm{N}_{2}$}

The same NMR sample of ${ }^{13} \mathrm{C}$-labeling study for $\mathbf{2 -}^{13} \mathbf{C}$ (Figure S15) was reused. Under $\mathrm{N}_{2}$, a brown solution of ${ }^{2-13} \mathbf{C}$ in benzene- $d_{6}$ was heated at $80{ }^{\circ} \mathrm{C}$. After 18 hours at $80{ }^{\circ} \mathrm{C},{ }^{1} \mathrm{H},{ }^{13} \mathrm{C}\left\{{ }^{1} \mathrm{H}\right\},{ }^{1} \mathrm{H}-{ }^{13} \mathrm{C}$ HSQC and ${ }^{1} \mathrm{H}_{-}^{13} \mathrm{C}$ HSQC HMBC NMR spectra were measured. The ${ }^{1} \mathrm{H}$ NMR spectrum (Figure S17) showed formation of $\mathbf{3 - 1}^{\mathbf{1 3}} \mathbf{C}$ as well as free $\mathrm{C}_{2} \mathrm{H}_{4}$ at $5.25 \mathrm{ppm}$. In the ${ }^{13} \mathrm{C}\left\{{ }^{1} \mathrm{H}\right\}$ NMR spectrum (Figure S18), two enriched ${ }^{13} \mathrm{C}$ NMR signals at $53.59 \mathrm{ppm}$ and $54.42 \mathrm{ppm}$ were observed as major species corresponding to two isotopologues of ${ }^{3-13} \mathrm{C}$, which have cross peaks $\delta{ }^{1} \mathrm{H}-{ }^{13} \mathrm{C}$ at $\delta-$ $0.28-53.59, \delta 1.07-53.59, \delta 1.44-54.42, \delta 4.34-54.42$ in the ${ }^{1} \mathrm{H}-{ }^{13} \mathrm{C}$ HSQC NMR spectra (Figure S19 and S20). Some minor ${ }^{13} \mathrm{C}$-enriched species were also observed at 50.79 ppm, $51.05 \mathrm{ppm}$ and $73.42 \mathrm{ppm}$ in the ${ }^{13} \mathrm{C}\left\{{ }^{1} \mathrm{H}\right\}$ NMR spectrum as well as in the ${ }^{1} \mathrm{H}-{ }^{13} \mathrm{C}$ HSQC and HMBC NMR spectra, but these species could not be characterized. 


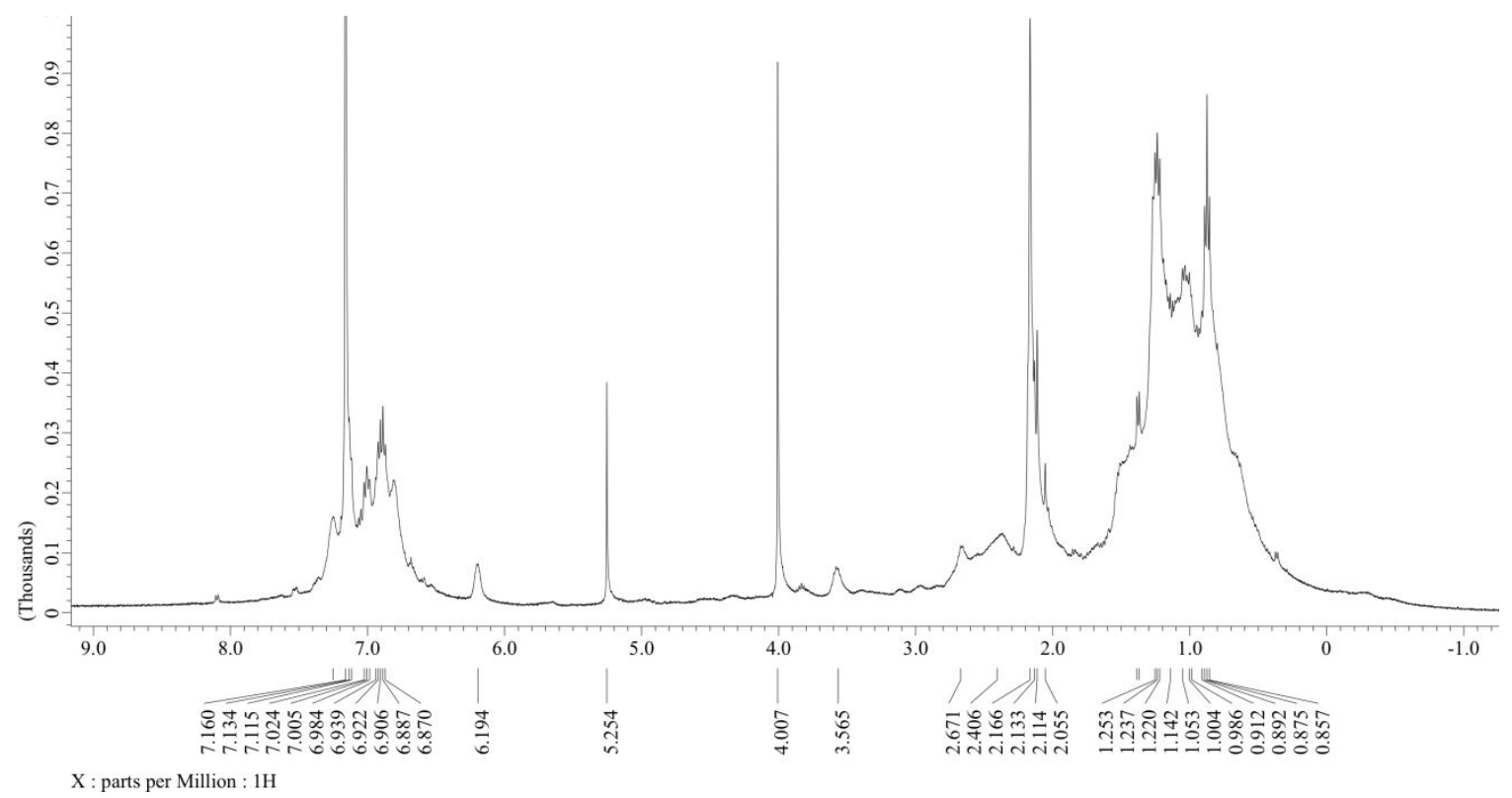

Figure S17. ${ }^{1} \mathrm{H}$ NMR spectrum of $2-{ }^{13} \mathbf{C}$ under $\mathrm{N}_{2}$ at $80{ }^{\circ} \mathrm{C}$ after 18 hours $(400 \mathrm{MHz}$, in Benzene- $d_{6}$, at $300 \mathrm{~K}$ ).

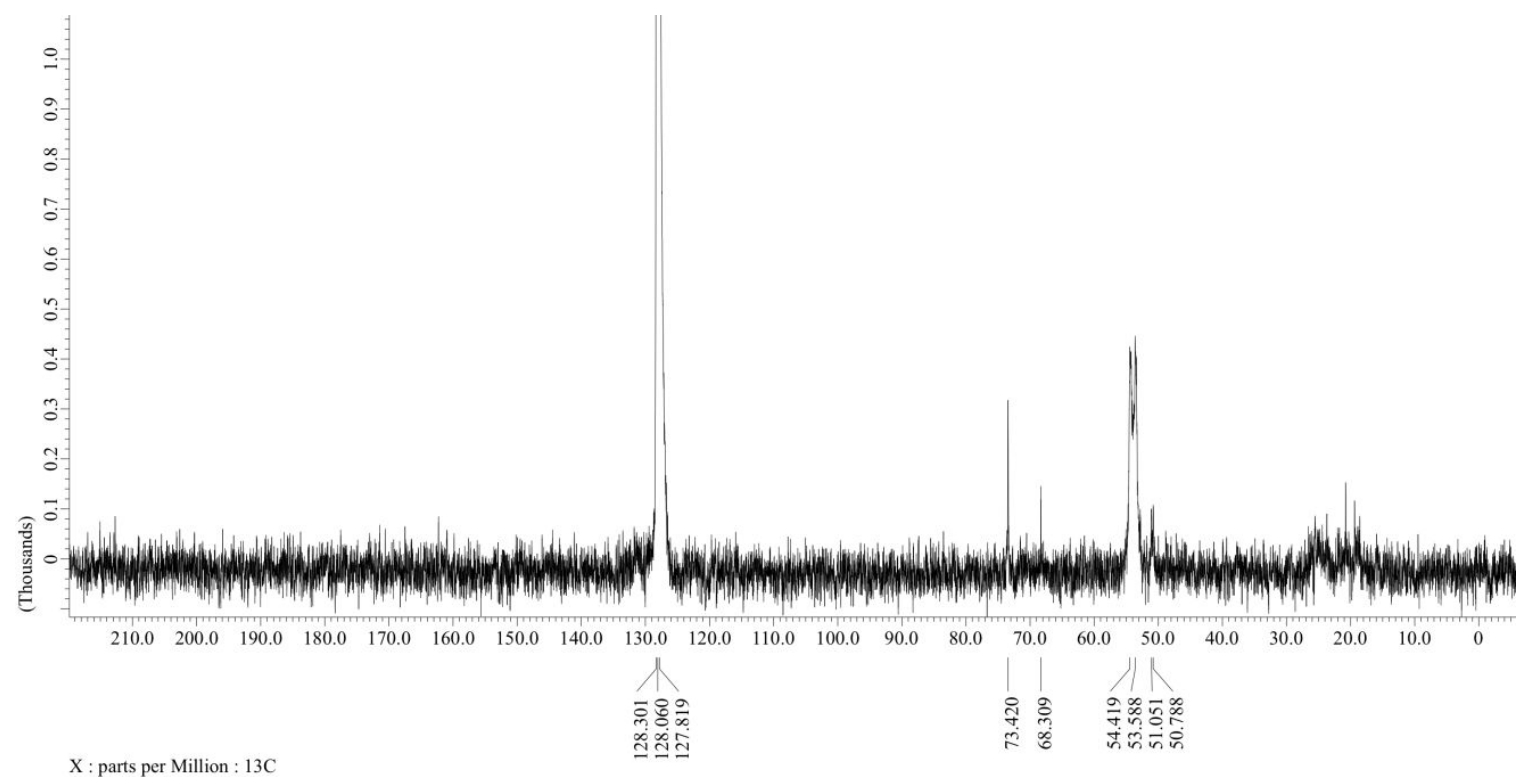

Figure S18. ${ }^{13} \mathrm{C}\left\{{ }^{1} \mathrm{H}\right\}$ NMR spectrum of ${ }^{2-13} \mathrm{C}$ under $\mathrm{N}_{2}$ at $80{ }^{\circ} \mathrm{C}$ after 18 hours $(101$ MHz, Benzene- $\left.d_{6}, 300 \mathrm{~K}\right)$. 


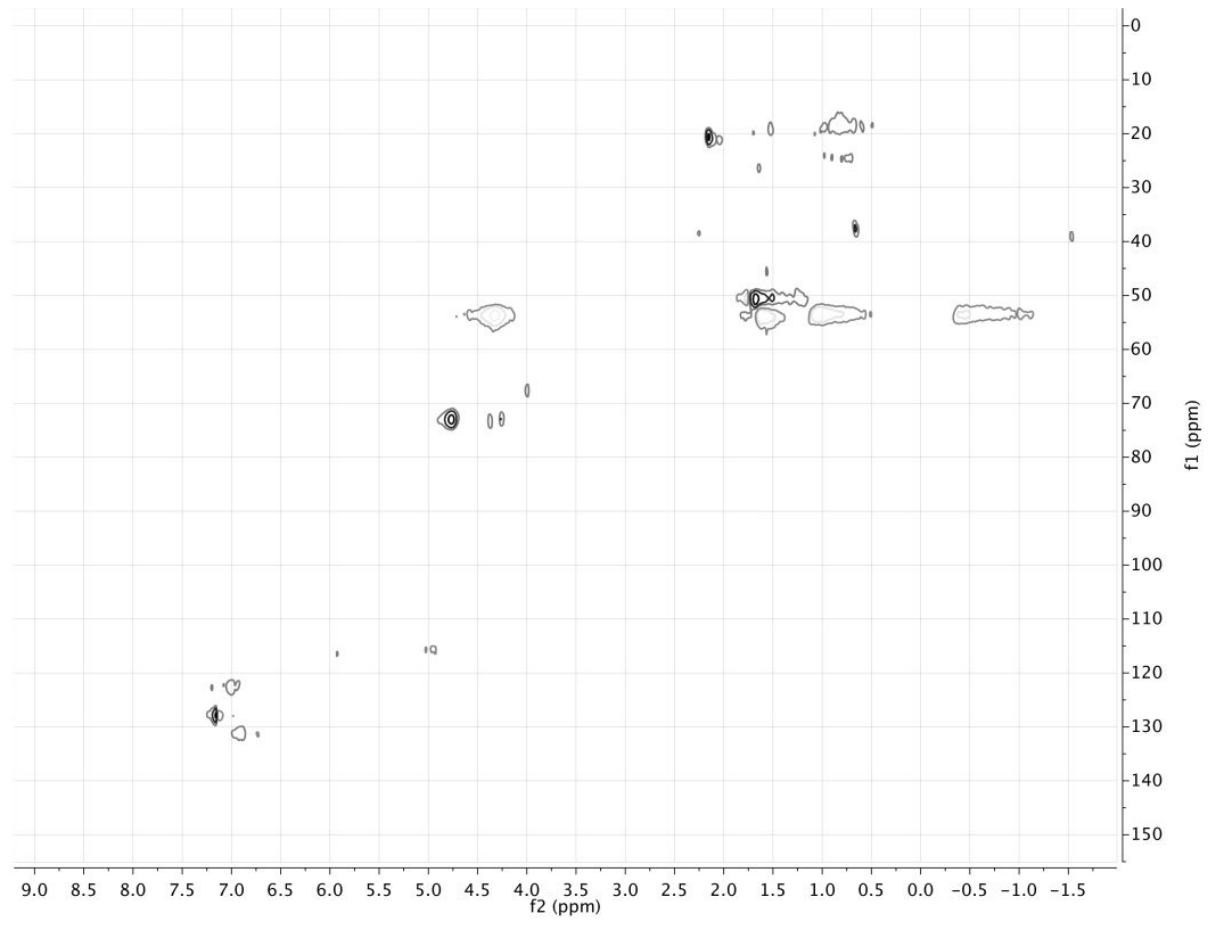

Figure S19. Decoupled ${ }^{1} \mathrm{H}-{ }^{13} \mathrm{C}$ HSQC NMR spectrum of $2-{ }^{13} \mathbf{C}$ under $\mathrm{N}_{2}$ at $80{ }^{\circ} \mathrm{C}$ after 18 hours (101 MHz, Benzene- $\left.d_{6}, 300 \mathrm{~K}\right)$.

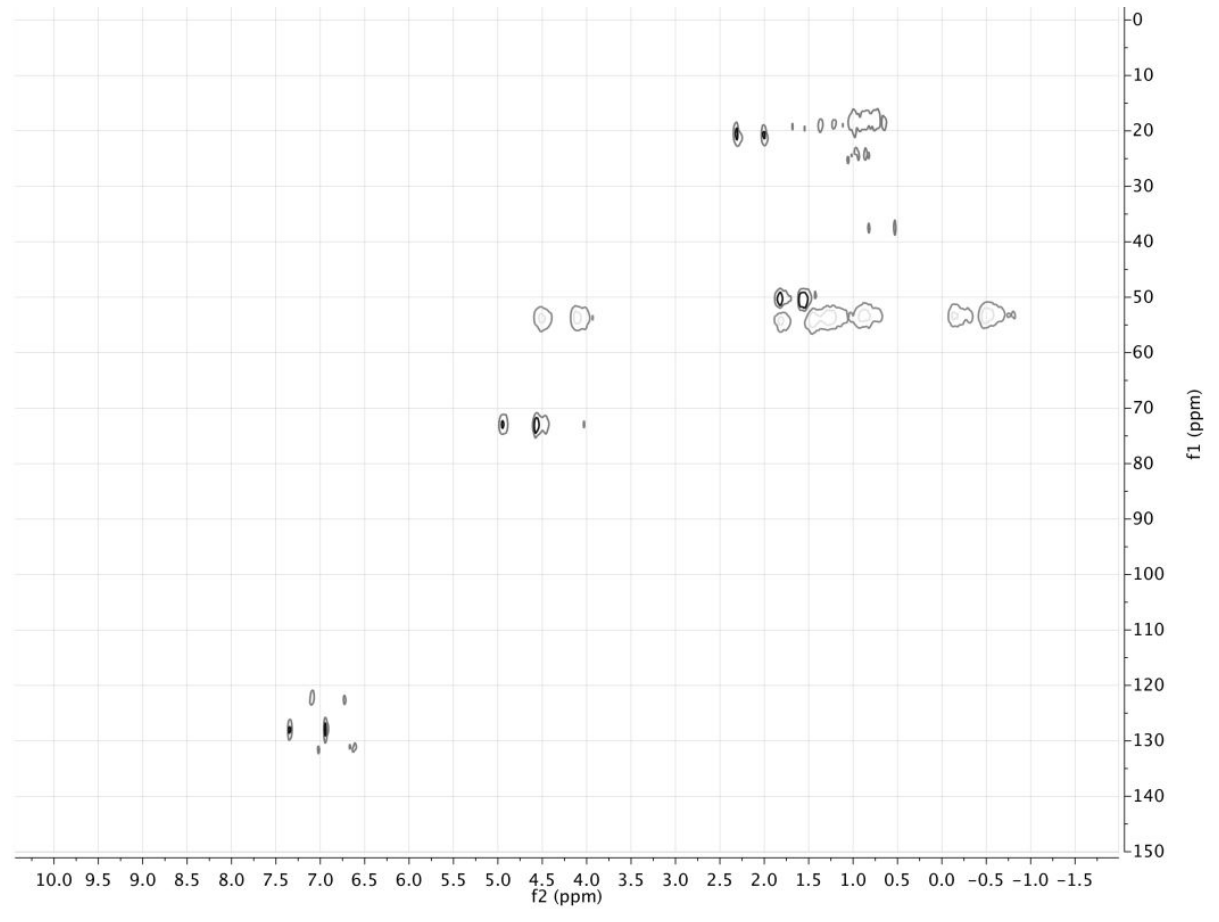

Figure S20. Non-decoupled ${ }^{1} \mathrm{H}-{ }^{13} \mathrm{C}$ HSQC NMR spectrum of $2-{ }^{13} \mathbf{C}$ under $\mathrm{N}_{2}$ at $80{ }^{\circ} \mathrm{C}$ after 18 hours (101 MHz, Benzene- $\left.d_{6}, 300 \mathrm{~K}\right)$. 


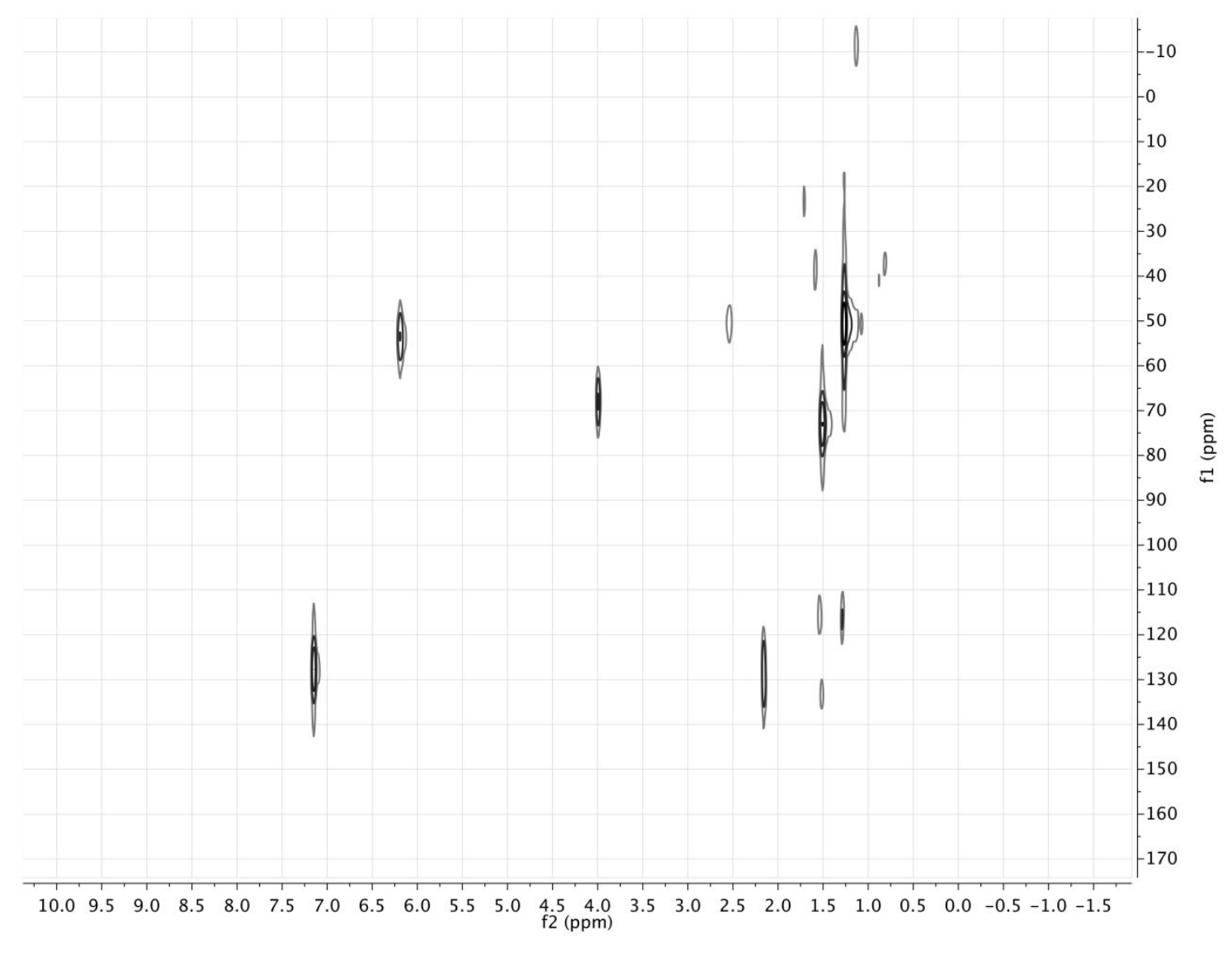

Figure S21. ${ }^{1} \mathrm{H}-{ }^{13} \mathrm{C}$ HMBC NMR spectrum of $2-{ }^{13} \mathbf{C}$ under $\mathrm{N}_{2}$ at $80{ }^{\circ} \mathrm{C}$ after 18 hours (101 MHz, Benzene- $\left.d_{6}, 300 \mathrm{~K}\right)$.

\section{Polymerization of 2-Norbornene by 1}

A $50 \mathrm{~mL}$ J-Young valve tube was charged with a green solution of 1 (15.2 mg, 21.4 $\mu \mathrm{mol})$ in toluene $(20 \mathrm{~mL})$ and 2-norbornene (200 mg, $2.12 \mathrm{mmol})$. The reaction mixture was heated at $80^{\circ} \mathrm{C}$, changing in color to brown. After stirring for 18 hours, the reaction mixture was poured into methanol $(30 \mathrm{~mL})$ with vigorous stirring, resulting in precipitation of a white solid. The white solid was collected by filtration, washed with methanol (10 mL x 2) and dried under vacuum to give a white solid (6.7 mg, 3\% yield). The yield of polynorbornene was irreproducible ranging in $3 \sim 10 \%$ yields. Multiple attempts to improve its yield by changing reaction temperature, time, concentration, ratio, and etc. were failed. In contrast to the reported cyclic polynorbornene by Veige, ${ }^{2}$ the obtained polynorbornene was poorly soluble in any organic solvents after 
precipitated. The NMR reaction of 2-norbornene with $\mathbf{1}$ in $\mathrm{C}_{6} \mathrm{D}_{6}$ revealed a mixture of cis- and trans-polynorbornenes in a 3:5 ratio, respectively, based on the integration ratio of the ${ }^{1} \mathrm{H}$ NMR spectrum (5.33 ppm and $\left.5.48 \mathrm{ppm}\right)$.

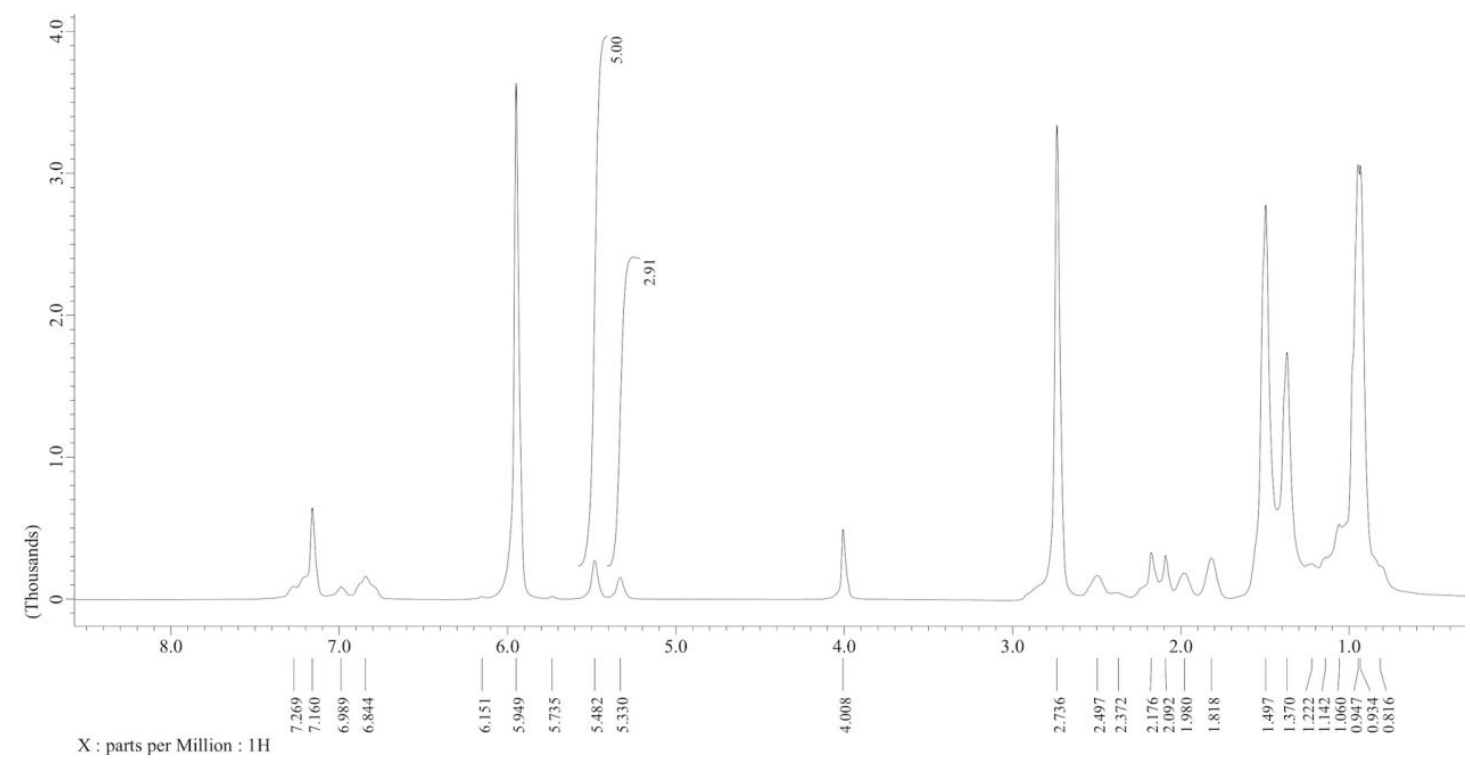

Figure S22. ${ }^{1} \mathrm{H}$ NMR spectrum of a mixture of $c i s$ - and trans-polynorbornenes resulted by 2-norbornene and 1 (400 MHz, in Benzene- $d_{6}$, at $\left.300 \mathrm{~K}\right)$.

\section{NMR Monitoring Reaction of $1-{ }^{13} \mathrm{C}$ with 2-Norbornene}

A $50 \mathrm{~mL}$ J-Young valve tube was charged with a green solution of $\mathbf{1 -}^{13} \mathrm{C} \cdot 0.5\left(\mathrm{FeCp}_{2}\right)$ (24.2 $\mathrm{mg}, 43.1 \mu \mathrm{mol})$ in toluene $(20 \mathrm{~mL})$ and 2-norbornene $(220 \mathrm{mg}, 2.34 \mathrm{mmol})$. The reaction mixture was heated at $80{ }^{\circ} \mathrm{C}$, changing in color to brown. After stirring for 18 hours, the reaction mixture was collected in to a J-Young valve NMR tube, ${ }^{1} \mathrm{H}$ and ${ }^{13} \mathrm{C}\left\{{ }^{1} \mathrm{H}\right\}$ NMR spectra were measured. The ${ }^{1} \mathrm{H}$ NMR spectrum showed a small doublet at $-0.71 \mathrm{ppm}(J=112 \mathrm{~Hz})$ along with cis- and trans-polynorbornenes at 5.23 and 5.44 ppm. The ${ }^{13} \mathrm{C}\left\{{ }^{1} \mathrm{H}\right\}$ NMR spectrum showed two broad signals at 58.8 and $59.0 \mathrm{ppm}$. 


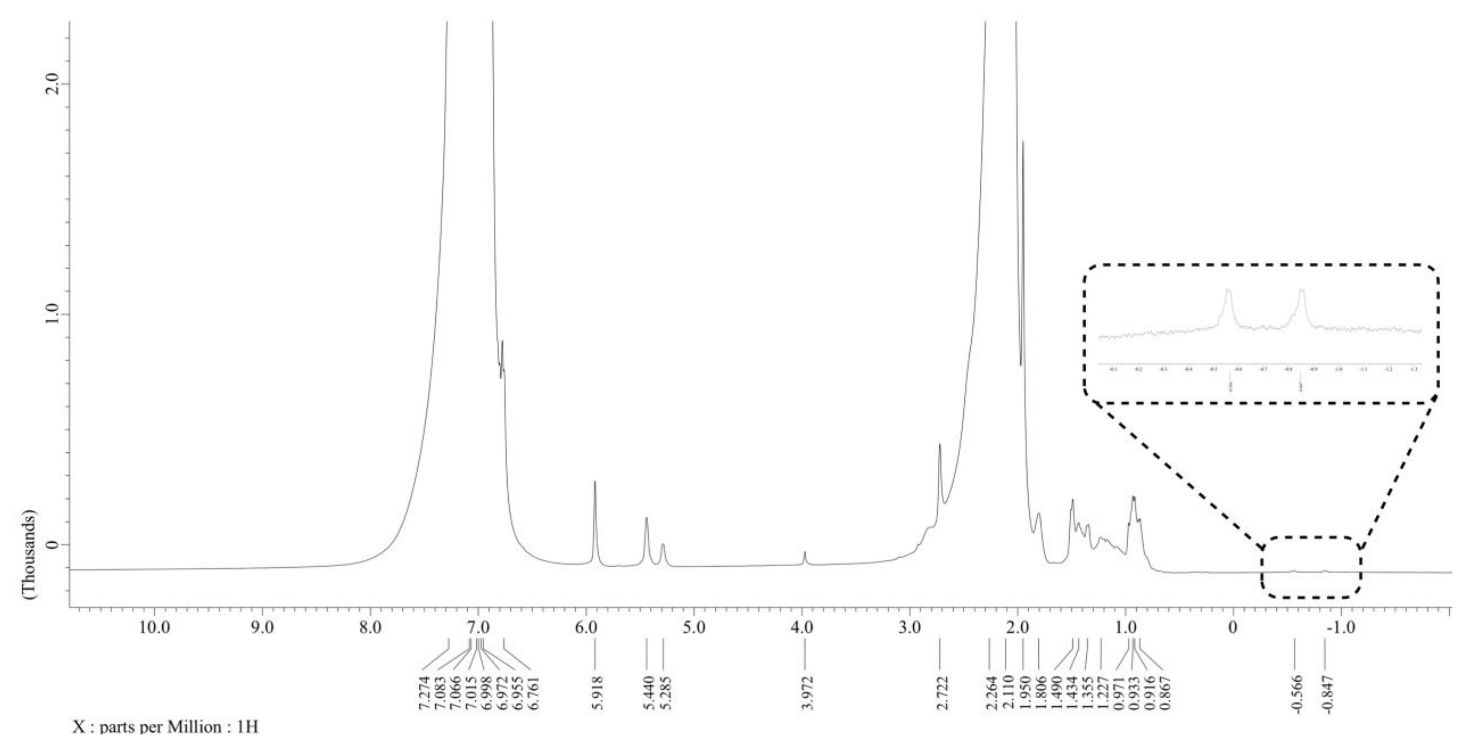

Figure S23. ${ }^{1} \mathrm{H}$ NMR spectrum of reaction of $\mathbf{1 -}^{\mathbf{1 3}} \mathbf{C}$ and 2-norbornene $(400 \mathrm{MHz}$, in toluene, at $300 \mathrm{~K})$.

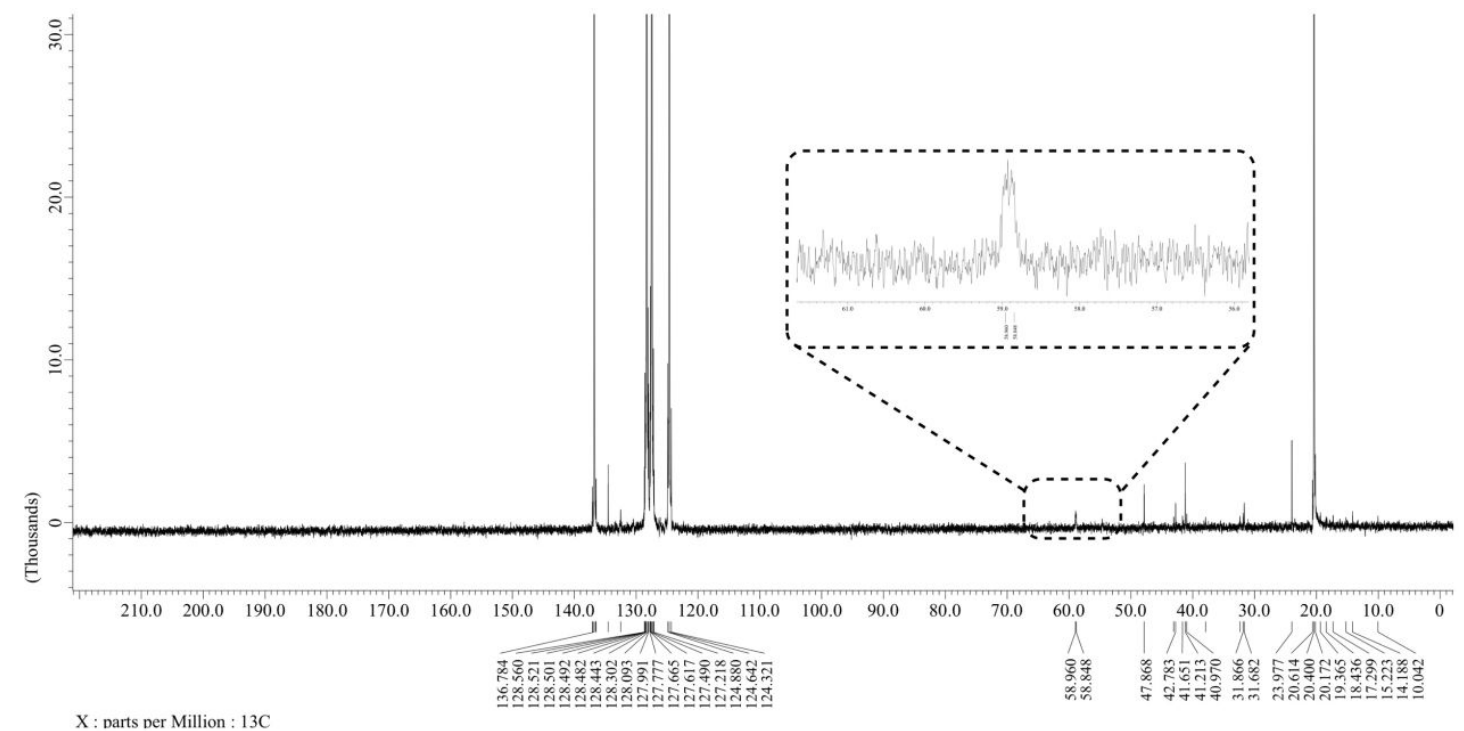

Figure S24. ${ }^{13} \mathrm{C}\left\{{ }^{1} \mathrm{H}\right\}$ NMR spectrum of reaction of $\mathbf{1 - 1 3} \mathbf{C}$ and 2-norbornene (101 MHz, in toluene, $300 \mathrm{~K})$. 


\section{X-ray Crystallography}

Crystallographic data are summarized in Table S1-S2. Suitable crystals for X-ray analyses of $\mathbf{2}$ and $\mathbf{3}$ were placed on the end of a Cryoloop coated in NVH oil. The Xray intensity data collection was carried out on a Bruker APEXII CCD area detector using graphite-monochromated Mo-K $\alpha$ radiation $(\lambda=0.71073 \AA)$ at $100(2) \mathrm{K}$. Preliminary indexing was performed from a series of thirty-six $0.5^{\circ}$ rotation frames with exposures of 10 seconds. Rotation frames were integrated using SAINT, ${ }^{3}$ producing a listing of non-averaged $F^{2}$ and $\sigma\left(F^{2}\right)$ values The intensity data were corrected for Lorentz and polarization effects and for absorption using SADABS. ${ }^{4}$ The initial structures were determined by the direct method on SHELXS. ${ }^{5}$ The further structure determination was performed by Fourier transform method and refined by least squares method on SHELXL. ${ }^{5,6}$ All reflections were used during refinement. Nonhydrogen atoms were refined anisotropically and hydrogen atoms were refined using riding models. For 2, one ${ }^{i} \operatorname{Pr}$ group of OAr was disordered over two positions and the thermal ellipsoids were fixed by SHELXL restraint commands. For 3, one of pentane molecules was located on a crystallographic special position and treated with PART-1. This pentane molecule was refined with $50 \%$ occupancy and the thermal ellipsoids were fixed by SHELXL restraint commands. These results were checked using the IUCR's CheckCIF routine. The alerts in the output are related to the disordered groups. 
Table S1. Summary of Structure Determination of 2

Empirical formula

Formula weight

Temperature

Wavelength

Crystal system

Space group

Cell constants:

$a$
$b$
$\alpha$

Volume

Z

Density (calculated)

Absorption coefficient

$\mathrm{F}(000)$

Crystal size

Theta range for data collection

Index ranges

Reflections collected

Independent reflections

Completeness to theta $=27.55^{\circ}$

Absorption correction

Max. and min. transmission

Refinement method

Data / restraints / parameters

Goodness-of-fit on $F^{2}$

Final $\mathrm{R}$ indices [I $>2 \operatorname{sigma}(\mathrm{I})]$

$\mathrm{R}$ indices (all data)

Largest diff. peak and hole
$\mathrm{C}_{43} \mathrm{H}_{66} \mathrm{NOP}_{2} \mathrm{Nb}$

767.81

100(2) K

$0.71073 \AA$

Monoclinic

$P 2_{1} / c$ (No. 14)

20.266(2) $\AA$

$11.5745(12) \AA$

18.789(2) $\AA$

$90^{\circ}$

$108.741(7)^{\circ}$

$90^{\circ}$

4173.5(8) $\AA^{3}$

4

$1.222 \mathrm{Mg} / \mathrm{m}^{3}$

$0.397 \mathrm{~mm}^{-1}$

1640

$0.20 \times 0.15 \times 0.05 \mathrm{~mm}^{3}$

1.061 to $27.650^{\circ}$

$-26 \leq \mathrm{h} \leq 26,-15 \leq \mathrm{k} \leq 15,-24 \leq 1 \leq 24$

81956

$9522[R($ int $)=0.0849]$

$97.8 \%$

Semi-empirical from equivalents

0.7456 and 0.6723

Full-matrix least-squares on $F^{2}$

9522 / 24 / 465

1.056

$R_{1}=0.0522, w R_{2}=0.1069$

$R_{1}=0.0979, w R_{2}=0.1272$

0.937 and -0.765 e. $\AA^{-3}$ 
Table S2. Summary of Structure Determination of 3·(pentane) ${ }_{1.5}$

Empirical formula

Formula weight

Temperature

Wavelength

Crystal system

Space group

Cell constants:

c

Volume

Z

Density (calculated)

Absorption coefficient

$\mathrm{F}(000)$

Crystal size

Theta range for data collection

Index ranges

Reflections collected

Independent reflections

Completeness to theta $=27.55^{\circ}$

Absorption correction

Max. and min. transmission

Refinement method

Data / restraints / parameters

Goodness-of-fit on $F^{2}$

Final R indices [I $>2 \operatorname{sigma}(\mathrm{I})]$

$\mathrm{R}$ indices (all data)

Largest diff. peak and hole
$\mathrm{C}_{48.5} \mathrm{H}_{80} \mathrm{NOP}_{2} \mathrm{Nb}$

847.98

100(2) K

$0.71073 \AA$

Triclinic

P-1 (No. 2)

12.107(2) $\AA$

14.193(3) ^

15.921(3) ^

$100.941(9)^{\circ}$

$103.252(9)^{\circ}$

$110.579(9)^{\circ}$

$2380.8(7) \AA^{3}$

2

$1.183 \mathrm{Mg} / \mathrm{m}^{3}$

$0.354 \mathrm{~mm}^{-1}$

914

$0.20 \times 0.12 \times 0.03 \mathrm{~mm}^{3}$

1.376 to $27.601^{\circ}$

$-15 \leq \mathrm{h} \leq 15,-18 \leq \mathrm{k} \leq 17,-20 \leq 1 \leq 20$

57955

$10750[R$ (int) $=0.0377]$

$97.3 \%$

Semi-empirical from equivalents

0.7456 and 0.6953

Full-matrix least-squares on $F^{2}$

10750 / 35 / 523

1.046

$R_{1}=0.0365, w R_{2}=0.0885$

$R_{1}=0.0472, w R_{2}=0.0939$

0.907 and -0.818 e. $\AA^{-3}$ 


\section{References}

(1) Kurogi, T.; Carroll, P. J.; Mindiola, D. J. J. Am. Chem. Soc. 2016, 138, 4306.

(2) Nadif, S. S.; Kubo, T.; Gonsales, S. A.; VenkatRamani, S.; Ghiviriga, I.; Sumerlin,

B. S.; Veige, A. S. J. Am. Chem. Soc. 2016, 138, 6408.

(3) SAINT; Bruker AXS Inc.: Madison, WI, USA. 2009.

(4) Sheldrick, G. M. SADABS; University of Gottingen: Germany, 2007.

(5) (a) Sheldrick, G. M. Acta Crystallogr. 2008, A64, 112. (b) Sheldrick, G. M. Acta Crystallogr. 2015, C71, 3.

(6) $R_{1}=\Sigma|| \mathrm{F}_{\mathrm{o}}|-| \mathrm{F}_{\mathrm{c}}|| / \Sigma\left|\mathrm{F}_{\mathrm{o}}\right|, w R_{2}=\left[\Sigma \mathrm{w}\left(\mathrm{F}_{\mathrm{o}}^{2}-\mathrm{F}_{\mathrm{c}}^{2}\right)^{2} / \Sigma \mathrm{w}\left(\mathrm{F}_{\mathrm{o}}^{2}\right)^{2}\right]^{1 / 2}, G O F=\left[\Sigma \mathrm{w}\left(\mathrm{F}_{\mathrm{o}}^{2}-\mathrm{F}_{\mathrm{c}}^{2}\right)^{2} /(\mathrm{n}\right.$

- $\mathrm{p})]^{1 / 2} ;$ where $\mathrm{n}=$ the number of reflections and $\mathrm{p}=$ the number of parameters refined. 